TRANSACTIONS OF THE

AMERICAN MATHEMATICAL SOCIETY

Volume 354, Number 11, Pages 4359-4391

S 0002-9947(02)03050-7

Article electronically published on July 8, 2002

\title{
ON THE REPRESENTATION THEORY OF LIE TRIPLE SYSTEMS
}

\author{
TERRELL L. HODGE AND BRIAN J. PARSHALL
}

\begin{abstract}
In this paper, we take a new look at the representation theory of Lie triple systems. We consider both ordinary Lie triple systems and restricted Lie triple systems in the sense of Hodge (2001). In a final section, we begin a study of the cohomology of Lie triple systems.
\end{abstract}

\section{INTRODUCTION}

A Lie triple system consists of a space $T$ of linear operators on a vector space $V$ that is closed under the ternary product $[x, y, z]=[[x, y], z]$, where $[x, y]=x y-y x$. Jacobson [15] first introduced them in connection with problems from Jordan theory and quantum mechanics, viewing Lie triple systems as subspaces of Lie algebras $\mathfrak{g}$ that are closed relative to the ternary product. (The two notions are equivalent.) For example, if $\theta$ is an involution (i.e., automorphism of order 2) of a Lie algebra $\mathfrak{g}$ over a field of characteristic $\neq 2$, the corresponding -1-eigenspace $T$ of $\theta$ is a Lie triple system in this sense. While the concept of a Lie triple system also has an abstract definition, all Lie triple systems have such realizations in terms of a Lie algebra and an involution. More recently, Lie triple systems have arisen in the study of symmetric spaces, e.g., [20], and have been connected with the study of the Yang-Baxter equations [18. Recently, Casas, Loday and Pirashvili 4 ] have generalized the notion of a Leibniz algebra to $n$-ary Leibniz algebras [4]; in the $n=3$ case, Lie triple systems form a subclass of these algebras.

This paper presents new results concerning the representation theory and homological algebra of Lie triple systems. We also include a development of these ideas for restricted Lie triple systems, introduced recently in 14. If $T$ is a Lie triple system of linear operators on the vector space $V$ defined over a field $k$ of positive characteristic $p>2$, then $T$ is restricted provided that $x^{p} \in T$ for all $x \in T$. Our original motivation came from the representation theory of algebraic groups, as we sketch below in connection with the results of Section 6 . However, the results presented here have an independent interest, and they are largely of a foundational nature. Beyond the connections with algebraic groups, another possible application of these results is to the theory of quasigroups and loops. For example, an algebraic commutative Moufang loop has a restricted Lie triple system as its tangent object

Received by the editors October 1, 2001 and, in revised form, March 18, 2002.

2000 Mathematics Subject Classification. Primary 17B99; Secondary 18G60, 20 G05.

Key words and phrases. Lie triple systems, restricted Lie triple systems, cohomology, representation theory.

Research supported in part by the National Science Foundation and a Research Development Award from Western Michigan University. 
22], and thus appropriate modules for such Lie triple systems should play a role in a representation theory of these loops.

We organize this paper as follows. Section 2 begins with the axiomatic definition of a Lie triple system $T$. In considering how $T$ arises as a subspace closed under the ternary product of a Lie algebra, we form the category $\mathbf{L I E}_{k}(T)$ of Lie algebras $\mathfrak{g}$ having a copy of $T$ as subspace closed under the ternary product and satisfying $\mathfrak{g} \cong T \oplus[T, T]$. We always assume that the underlying field $k$ has characteristic $\neq 2$; so $\mathfrak{g}$ clearly has an involution $\theta$ in which $T$ identifies with the -1-eigenspace. The category $\mathbf{L I E}_{k}(T)$ contains both an initial object $L_{u}(T)$ and a terminal object $L_{s}(T)$; both of these algebras play an important role in the representation theory of $T$.

Section 3 takes up the category $T$-Mod of $T$-module $\$ 1$ and its precise relationship to the category $\left(L_{u}(T), \theta\right)$-Mod, consisting of modules for the Lie algebra $L_{u}(T)$ that have a compatible action of $\theta$. The functor $j^{*}:\left(L_{u}(T), \theta\right)-\mathbf{M o d} \rightarrow$ $T$-Mod that assigns to each $\left(L_{u}(T), \theta\right)$-module $V$ the $T$-module $j^{*} V$ obtained by taking the -1-eigenspace of $\theta$ on $V$ fits into the diagram

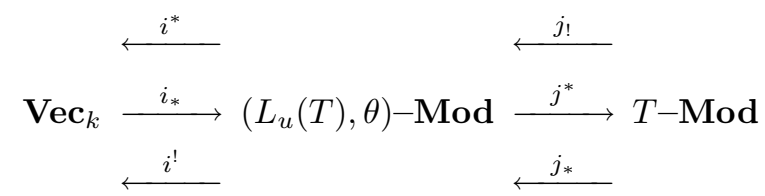

in which $\left(i^{*}, i_{*}, i^{!}\right)$and $\left(j_{!}, j^{*}, j_{*}\right)$ are adjoint triples. Here $\mathbf{V e c}_{k}$ is the category of vector spaces over $k$. In essence, Section 3 consists of a detailed exploitation of (1.1), reminiscent of, but considerably more elementary than, the use of "recollement diagrams" for triangulated categories developed in 11. For example, we prove $T$-Mod is a quotient category of $\left(L_{u}(T), \theta\right)-\mathbf{M o d}$, show that $T$-Mod has enough injective and projective objects, characterize the "standard extension" (as defined in [12]) of a $T$-module in terms of the functor $j^{*}$, and prove that $T$-Mod is isomorphic to the category of modules for the algebra $U(T)^{\theta}$ of fixed points of $\theta$ in the universal enveloping algebra $U(T)=U\left(L_{u}(T)\right)$ of $L_{u}(T)$. We point out that, in many interesting cases, the Lie algebra $L_{u}(T)$ can be explicitly identified; see [14. However, we know of no systematic study of the representation theory of $U(T)^{\theta}$.

Section 5 parallels Section 3 by showing how the results there extend to the context of restricted representations for restricted Lie triple systems. In preparation for this work, Section 4 begins with a recasting of some basic results of [14] on the general theory of restricted Lie triple systems. For a restricted Lie triple system $T$, we then introduce the category $\mathbf{L I E}_{k}^{[p]}(T)$, whose objects are those Lie algebras $\mathfrak{g} \in \mathbf{L I E}_{k}(T)$ satisfying, for each $a \in T \subset \mathfrak{g},(\operatorname{ad} a)^{p}=\operatorname{ad} a^{[p]}$ for some $a^{[p]} \in T$. Such Lie algebras are not necessarily restricted Lie algebras. We determine initial and terminal objects $L_{u}^{[p]}(T)$ and $L_{s}^{[p]}(T)$ for $\mathbf{L I E}_{k}^{[p]}(T)$, introduce an associative algebra analogue $U^{[p]}(T)$ (with involution $\theta$ ) of the restricted enveloping algebra of a restricted Lie algebra, and obtain an equivalence between an appropriate module category $\left(L_{u}^{[p]}(T), \theta\right)_{[p]}$-Mod and the module category $\left(U^{[p]}(T), \theta\right)$-Mod. These module categories then reappear in Section 5 , where we consider restricted modules and establish relationships akin to those of (1.1), for example.

\footnotetext{
${ }^{1}$ Our definition of a $T$-module comes from Harris [12]. There are alternative notions of a module for a Lie triple system $T$; see (3.18 for a discussion.
} 
Given a restricted Lie algebra $\mathfrak{g}$ over a field $k$ of positive characteristic $p>2$, there exist well-known connections between the restricted cohomology of $\mathfrak{g}$ and the restricted nullcone $\mathcal{N}_{1}(\mathfrak{g})$, consisting of all $X \in \mathfrak{g}$ satisfying $X^{[p]}=0$. The full nullcone $\mathcal{N}(\mathfrak{g})$ consists of all $[p]$-nilpotent elements in $\mathfrak{g}$, and $\mathcal{N}_{1}(\mathfrak{g})$ is a closed subvariety of it. To each finite-dimensional, restricted $\mathfrak{g}$-module $M$ there is associated a closed, conical subvariety $V_{\mathfrak{g}}(M)$ of $\mathcal{N}_{1}(\mathfrak{g})$, called its support variety, which carries information concerning the cohomology of $M$. See [8], 9], 27], 28]. In addition, as shown in [23, cohomological methods provide powerful tools for studying the structure of $\mathcal{N}_{1}(\mathfrak{g})$. This paper has its original motivation in our efforts to generalize this theory to the case of restricted Lie triple sytems. Here the most interesting cases occur when the Lie triple system $T=\mathfrak{p} \subset \mathfrak{g}=\operatorname{Lie}(G)$ arises from an involution on a reductive algebraic group $G$. In this case, Richardson 25] has provided considerable information on the structure of the unipotent cone in the associated symmetric space, isomorphic to $\mathcal{N}(\mathfrak{p})$ when $p$ is a good prime for $G$.

Although we have much yet to do towards accomplishing this desired generalization to Lie triple system theory, Section 6 does provide the beginnings of a cohomology theory for Lie triple systems and their restricted versions. Toward that end, it begins by reviewing and extending some previous results by Harris 12] concerning cohomology in the category $\left(L_{u}(T), \theta\right)$-Mod, for a given Lie triple system $T$. The cohomology theory for $T$-Mod appears to be considerably more subtle. Our general approach again exploits the diagram (1.1). For example, we provide a spectral sequence relating the cohomology in $\left(L_{u}(T), \theta\right)$-Mod to that in $T$-Mod. Unlike the case for a finite-dimensional Lie algebra, objects in $T$-Mod need not have finite homological dimension. Finally, we consider a natural generalization of the Hochschild map (see, e.g., [10]) to the context of restricted Lie triple sytems. We note that, although our notion of a module for a Lie triple $T$ system yields a representation for $T$ as a 3-ary Leibniz algebra in the sense of [4] (resp., a module for $T$ in the sense of Yamaguti [29]), our theory of cohomology for Lie triple systems differs from the cohomology of 3-ary Liebniz algebras defined in [4] (resp., from the cohomology as defined in 29]).

We thank Nora Hopkins, Michael Kinyon, and Bob Stong for a number of helpful discussions. We are also grateful for the referee's sharp eyes and thoughtful remarks.

\section{LIE TRIPLE SYSTEMS}

Throughout this paper, $k$ will be an algebraically closed field. A standing assumption will be that $k$ has characteristic not equal to 2. Unless otherwise stated, all vector spaces, algebras, tensor products, etc., will be taken over $k$. We begin by reminding the reader of some basic definitions.

Definition 2.1. A Lie triple system is a vector space $T$ equipped with a trilinear ternary product $[a, b, c]$ satisfying the following three properties $(a, b, c, x, y, z \in T)$ :

$$
\begin{gathered}
{[a, a, b]=0} \\
{[a, b, c]+[b, c, a]+[c, a, b]=0} \\
{[a, b,[x, y, z]]=[[a, b, x], y, z]+[x,[a, b, y], z]+[x, y,[a, b, z]] .}
\end{gathered}
$$

A morphism $\phi: T \rightarrow T^{\prime}$ of Lie triple systems is a linear map preserving the triple product. Let $\mathbf{L T S}_{k}$ denote the category of Lie triple systems over $k$. 
If $T \in \mathbf{L T S}_{k}$, then (2.1a) implies that $0=[(a+b),(a+b), c]=[a, a, c]+[a, b, c]+$ $[b, a, c]+[b, b, c]=[a, b, c]+[b, a, c]$, so that

$$
[a, b, c]=-[b, a, c]
$$

This simple observation perhaps suggests how Lie triple systems arise from Lie algebras. Given a Lie algebra $\mathfrak{g}$ over $k$, the triple product on $\mathfrak{g}$ given by $[a, b, c]=[[a, b], c]$ for $a, b, c \in \mathfrak{g}$ satisfies the conditions of (2.1). Let $\mathfrak{g}_{\text {trip }}$ denote the corresponding Lie triple system. Second, if $\theta: \mathfrak{g} \rightarrow \mathfrak{g}$ is an involutory automorphism, the -1eigenspace $T \subseteq \mathfrak{g}$ of $\theta$ is a Lie triple system which is a subsystem of $\mathfrak{g}_{\text {trip }}$.

A derivation of a Lie triple system $T$ is a linear map $D: T \rightarrow T$ satisfying $D([a, b, c])=[D(a), b, c]+[a, D(b), c]+[a, b, D(c)]$ for $a, b, c \in T$. The space $\operatorname{Der}(T)$ of all derivations of $T$ has a natural Lie algebra structure, given by setting $[D, E]=$ $D E-E D$. For $a, b \in T$, consider the inner derivation $D_{a, b} \in \operatorname{Der}(T)$ given by $D_{a, b}(c)=[a, b, c], c \in T$. For any $D \in \operatorname{Der}(T)$ and $a, b \in T$,

$$
\left[D, D_{a, b}\right]=D_{D(a), b}+D_{a, D(b)} \text {. }
$$

The space $T \oplus \operatorname{Der}(T)$ also has a natural Lie algebra structure, defined by setting

$$
[(a, D),(b, E)]=\left(D(b)-E(a), D_{a, b}+[D, E]\right)
$$

for $(a, D),(b, E) \in T \oplus \operatorname{Der}(T)$. Furthermore,

$$
\theta: T \oplus \operatorname{Der}(T) \rightarrow T \oplus \operatorname{Der}(T), \quad(a, D) \mapsto(-a, D),
$$

is an involutory Lie algebra automorphism having $T$ as the -1-eigenspace. Thus, any Lie triple system $T$ is a Lie triple subsystem of some $\mathfrak{g}_{\text {trip }}$, e.g., take $\mathfrak{g}=$ $T \oplus \operatorname{Der}(T)$.

If $\phi: T \rightarrow \mathfrak{g}_{\text {trip }}$ is a morphism of Lie triple systems, let $\mathfrak{g}_{\phi}=\phi(T)+[\phi(T), \phi(T)]$ denote the Lie subalgebra of $\mathfrak{g}$ generated by the image of $\phi$. Let $\mathbf{L} \mathbf{I E}_{k}$ be the category of Lie algebras over $k$. Fix a Lie triple system $T$, and let $\mathbf{L I E}_{k}(T)$ be the category of triples $(\mathfrak{g}, \widetilde{T}, \phi)$ satisfying the following conditions: (a) $\mathfrak{g} \in \mathbf{L I E}_{k}$; (b) $\widetilde{T}$ is a Lie triple subsystem of $\mathfrak{g}_{\text {trip }}$ such that $\mathfrak{g}=\widetilde{T} \oplus[\widetilde{T}, \widetilde{T}]$; and (c) $\phi: T \stackrel{\sim}{\rightarrow} \widetilde{T}$ is an isomorphism of Lie triple systems. Thus, $\mathfrak{g}=\mathfrak{g}_{\phi}$. A morphism $(\mathfrak{g}, \widetilde{T}, \phi) \rightarrow$ $\left(\mathfrak{g}^{\prime}, \widetilde{T}^{\prime}, \phi^{\prime}\right)$ is given by a Lie algebra morphism $\Psi: \mathfrak{g} \rightarrow \mathfrak{g}^{\prime}$ such that $\left.\Psi\right|_{\widetilde{T}} \circ \phi=\phi^{\prime}$. Observe that for any $(\mathfrak{g}, \widetilde{T}, \phi) \in \mathbf{L I E}_{k}(T), \mathfrak{g}$ is a $\mathbb{Z}_{2}$-graded Lie algebra

$$
\mathfrak{g}=\mathfrak{g}_{1} \oplus \mathfrak{g}_{0}
$$

where $\mathfrak{g}_{1}=\widetilde{T}$ and $\mathfrak{g}_{0}=[\widetilde{T}, \widetilde{T}]$.

The category $\mathbf{L I E}_{k}(T)$ contains a terminal object. Define $L_{s}(T)$ to be the Lie subalgebra of $T \oplus \operatorname{Der}(T)$ generated by $T 2$ Thus,

$$
L_{s}(T)=T \oplus \operatorname{InnDer}(T),
$$

where $\operatorname{InnDer}(T):=\operatorname{Span}\left\{D_{a, b} \mid a, b \in T\right\}$ is the subspace of $\operatorname{Der}(T)$ spanned by the inner derivations. Observe that $\operatorname{InnDer}(T)=[T, T]$ is a subalgebra of $L_{s}(T)$. Now consider $\left(L_{s}(T), T\right.$, id $) \in \mathbf{L I E}_{k}(T)$ and let $(\mathfrak{g}, \widetilde{T}, \phi) \in \mathbf{L I E}_{k}(T)$. Extend $\phi^{-1}$ : $\widetilde{T} \rightarrow T$ to a linear map $\Phi^{\prime}: \widetilde{T} \otimes \widetilde{T} \rightarrow L_{s}(T)$ by putting $\Phi^{\prime}(a \otimes b)=D_{\phi^{-1}(a), \phi^{-1}(b)}$. If $\sum a_{i} \otimes b_{i} \in \widetilde{T} \otimes \widetilde{T}$ satisfies $\sum\left[a_{i}, b_{i}\right]=0$ in $\mathfrak{g}$, then $0=\sum\left[\left[\phi^{-1}\left(a_{i}\right), \phi^{-1}\left(b_{i}\right)\right], x\right]=$ $\sum\left[\phi^{-1}\left(a_{i}\right), \phi^{-1}\left(b_{i}\right), x\right]$ for all $x \in T$. Hence $\sum D_{\phi^{-1}\left(a_{i}\right), \phi^{-1}\left(b_{i}\right)}=0$; so $\phi^{-1}$ and

\footnotetext{
${ }^{2}$ The definitions imply that $L_{s}(T) \cong T \oplus(T \otimes T / I)$, where $I$ is the subspace of $T \otimes T$ consisting of elements $\sum a_{i} \otimes b_{i}$ satisfying $\sum\left[a_{i}, b_{i}, x\right]=0$ for all $x \in T$. In fact, $L_{s}(T)$ may be defined simply by setting up the appropriate bracket relations on the space $T \oplus(T \otimes T / I)$; see e.g., [15].
} 
$\Phi^{\prime}$ define a unique linear map $\Phi: \mathfrak{g} \rightarrow L_{s}(T)$ which is necessarily a Lie algebra morphism. It follows that there is a unique morphism $(\mathfrak{g}, \widetilde{T}, \phi) \rightarrow\left(L_{s}(T), T\right.$, id $)$ in $\mathbf{L I E}_{k}(T)$, so that $\left(L_{s}(T), T\right.$, id $)$ is the required terminal object.

In addition, $\mathbf{L I E}_{k}(T)$ has an initial object $\left(L_{u}(T), T\right.$,id $)$. Let $\mathfrak{L}$ be the free Lie algebra based on $T$, and put $L_{u}(T)=\mathfrak{L} / \mathfrak{I}$, where $\mathfrak{I}$ is the ideal in $\mathfrak{L}$ generated by the elements $[a, b, c]-[[a, b], c], a, b, c \in T$. If $\iota: T \rightarrow L_{u}(T)$ is the composition $T \rightarrow \mathfrak{L} \rightarrow L_{u}(T)$, then, by construction, for any Lie algebra $\mathfrak{g}$ and any morphism $\psi: T \rightarrow \mathfrak{g}_{\text {trip }}$, there exists a unique Lie algebra morphism $\Phi: L_{u}(T) \rightarrow \mathfrak{g}$ such that $\psi=\Phi \circ \iota$. In particular, taking $\mathfrak{g}=L_{s}(T)$, we conclude that $L_{u}(T)=T \oplus[T, T]$; so, $L_{u}(T)=L_{u}(T)_{\iota}$ and $\iota=$ id. Obviously, $\left(L_{u}(T), T\right.$, id $) \in \mathbf{L I E}_{k}(T)$ is an initial object in $\operatorname{LIE}_{k}(T)$ We will let $\theta: L_{u}(T) \rightarrow L_{u}(T)$ be the involution with -1eigenspace $T$.

If $U(T)$ denotes the universal enveloping algebra $U\left(L_{u}(T)\right)$ of the Lie algebra $L_{u}(T)$, any linear map $\phi: T \rightarrow A$ from $T$ into an associative algebra $A$ satisfying $\phi([a, b, c])=[[\phi(a), \phi(b)], \phi(c)]$ clearly extends uniquely to a morphism $U(T) \rightarrow A$ of associative algebras. In fact, one may define the universal associative algebra of $T$ independently of $L_{u}(T)$ as the associative algebra that satisfies the universal property indicated above. One can also construct this algebra by taking the quotient $\mathfrak{F} / \mathfrak{J}$ of the free associative algebra $\mathfrak{F}$ on $T$ by the ideal $\mathfrak{J}$ generated by the elements $[a, b, c]-[[a, b], c], a, b, c \in T$, and then check that it is isomorphic to $U(T)$.

Example 2.4. Let $G$ be an affine algebraic group over $k$, and assume that $\theta: G \rightarrow$ $G$ is an involution of algebraic groups. Let $\theta$ also denote the induced involution on the Lie algebra $\mathfrak{g}$ of $G$. In this situation, the -1-eigenspace of $\theta$ on $\mathfrak{g}$ will be denoted $\mathfrak{p}$. It is a Lie triple subsystem of $\mathfrak{g}_{\text {trip }}$.

Assume that $G$ is a simple, simply connected group. In case $k$ has characteristic 0 , it follows easily from [16, 7.3] that $L_{u}(\mathfrak{p}) \cong L_{s}(\mathfrak{p}) \cong \mathfrak{g}$. When $k$ has positive characteristic $p$, the Lie algebras $L_{u}(\mathfrak{p})$ and $L_{s}(\mathfrak{p})$ have all been determined precisely in [14, 4.21]. For example, if $p>3$, then $L_{u}(\mathfrak{p})=\mathfrak{g}$ in all cases. Also, $L_{s}(\mathfrak{p})=\mathfrak{g}$ except when $G$ has type $A_{n}, n>2$ and $p \mid(n+1)$. In that case, $L_{s}(\mathfrak{p})=\mathfrak{g} / Z(\mathfrak{g})$.

Example 2.5. Call a Lie triple system $T$ abelian if $[a, b, c]=0$ for all $a, b, c \in T$. When $T$ is abelian, $L_{s}(T)$ identifies with the abelian Lie algebra having underlying vector space $T$. Also, $L_{u}(T)=T \oplus \bigwedge^{2} T$ with $[x, y]=x \wedge y$ for $x, y \in T$ and with all other products vanishing.

We now introduce some useful notation. Suppose that $A$ is an associative algebra with involution $\theta$. We form a new algebra $K(A, \theta)=A \oplus A$ with multiplication given by

$$
(a, b)(c, d)=(a c+b \theta(d), b \theta(c)+a d) .
$$

It will be convenient to denote $(a, b)$ by $a+b \theta$ (and thus $K(A, \theta)$ by $A \oplus A \theta$ ). Suppose that $A$ has an augmentation $\epsilon: A \rightarrow k$ satisfying $\epsilon \theta(a)=\epsilon(a)$ for all $a \in A$. Define $\epsilon^{+}: K(A, \theta) \rightarrow k$ (resp., $\epsilon^{-}: K(A, \theta) \rightarrow k$ ) by $\epsilon^{+}(a+b \theta)=\epsilon(a+b)$ (resp., $\epsilon^{-}(a+b \theta)=\epsilon(a-b)$ ). Both $\epsilon^{+}$and $\epsilon^{-}$are algebra homomorphisms. The field $k$ regarded as a $K(A, \theta)$-module by means of $\epsilon^{+}$(resp., $\epsilon^{-}$) will usually be denoted $k^{+}$(resp., $\left.k^{-}\right)$. Any direct sum of copies of $k^{+}$will be called a trivial module for $K(A, \theta)$.

\footnotetext{
${ }^{3}$ Traditionally, the Lie algebra $L_{s}(T)$ (resp., $L_{u}(T)$ ) is known as the standard (enveloping) Lie algebra (resp., universal (enveloping) Lie algebra) of $T$. Although these objects are not new, our approach to them via terminal (resp., initial) objects in an appropriately defined category is.
} 
As an example, we may take $A=U(T)$, since the natural involution $\theta$ on $L_{u}(T)$ extends to an involution, still denoted $\theta$, on $U(T)$. Thus, we can form the algebra $K(T):=K(U(T), \theta)$, which will play an important role in the next section. To close this section, we record one more fact about the structure of $K(A, \theta)$ for $A$ arbitrary, and discuss its implications for the special case $A=U(T)$.

Lemma 2.6. Let $A$ be an associative algebra with involution $\theta$. Suppose $A$ is a Hopf algebra, with counit $\epsilon$, comultiplication $\Delta$, and antipode $\gamma$. Suppose also that $\theta$ is an involution of Hopf algebras, i.e., $\Delta \circ \theta=(\theta \otimes \theta) \circ \Delta, \epsilon \circ \theta=\epsilon$, and $\gamma \circ \theta=\theta \circ \gamma$. Then this structure induces a Hopf algebra structure on $K(A, \theta)$ with comultiplication $\Delta_{K}$ satisfying $\Delta_{K}(\theta)=\theta \otimes \theta$, counit $\epsilon^{+}$satisfying $\epsilon^{+}(\theta)=1$, and antipode $\gamma_{K}$ satisfying $\gamma_{K}(\theta)=\theta$.

Proof. Define $\Delta_{K}: K(A, \theta) \rightarrow K(A, \theta) \otimes K(A, \theta)$ by $\Delta_{K}(a+b \theta)=\Delta(a)+\Delta(b)(\theta \otimes$ $\theta)$. One may verify directly that this map is a cocommutative algebra morphism satisfying the coassociative axiom $\left(1 \otimes \Delta_{K}\right) \Delta_{K}=\left(\Delta_{K} \otimes 1\right) \Delta_{K}$. We have already seen that the counit $\epsilon: A \rightarrow k$ extends to an algebra morphism $\epsilon^{+}: K(A, \theta) \rightarrow k$. Then $\left(\epsilon^{+} \otimes 1\right) \Delta_{K}=\left(1 \otimes \epsilon^{+}\right) \Delta_{K}$ is the identity map on $K(A, \theta)$. Finally, the antipode $\gamma$ on $A$ extends to an antipode $\gamma_{K}: K(A, \theta) \rightarrow K(A, \theta)$ by putting $\gamma_{K}(a+b \theta)=\gamma(a)+\theta \gamma(b)=\gamma(a)+\gamma(\theta(b)) \theta$.

In particular, let $A=U(T)$ again. Since $U(T)$ is the universal enveloping algebra of the Lie algebra $L_{u}(T), U(T)$ has a (cocommutative) Hopf algebra structure, with comultiplication $\Delta$ defined from the map $L_{u}(T) \rightarrow U(T) \otimes U(T), x \mapsto x \otimes 1+1 \otimes x$, and antipode $\gamma$ determined by $\gamma(x)=-x$ for (the primitive elements) $x \in L_{u}(T)$. Furthermore, the induced involution $\theta$ on $U(T)$ is a Hopf algebra involution. Thus, $K(T)$ is also a (cocommutative) Hopf algebra by (2.6).

\section{Modules FOR Lie TRIPLE Systems}

Although a module $V$ for a Lie algebra $\mathfrak{g}$ over $k$ is perhaps most succinctly defined via a Lie algebra homomorphism $\xi: \mathfrak{g} \rightarrow \mathfrak{g l}(V)$, an equivalent formulation says that a vector space $V$ is a module for $\mathfrak{g}$ if there is a Lie algebra structure on $\mathfrak{g}_{V}:=\mathfrak{g} \oplus V$ for which (a) $\mathfrak{g}$ is a subalgebra of $\mathfrak{g}_{V}$; (b) $V$ is an ideal, i.e., for $x, y \in \mathfrak{g} \oplus V ;[x, y] \in V$ if one of $x, y \in V ;$ and (c) $[x, y]=0$ if both $x, y \in V$. Note that for $x \in \mathfrak{g}$ and $y \in V$, defining $\xi(x)(y)=[x, y]$ recovers the corresponding homomorphism $\xi: \mathfrak{g} \rightarrow \mathfrak{g l}(V)$. Consequently, writing $x . y$ for $\xi(x)(y)$ as usual, we shall freely identify the expression $x . y$ with $[x, y]$. Now let $T$ be a Lie triple system over $k$. In a similar way, and following [12], we say that a vector space $M$ is a $T$ module provided that $E_{M}:=T \oplus M$ possesses the structure of a Lie triple system such that: (a) $T$ is a Lie triple subsystem of $E_{M}$, (b) for $a, b, c \in E_{M},[a, b, c] \in M$ if any one of $a, b, c$ lies in $M$, and (c) $[a, b, c]=0$ if any two of $a, b, c$ lie in $M$. We note that a subspace $A$ of a Lie triple system $T$ is called an ideal if $[A, T, T] \subset A$. Condition (b) is thus equivalent to the statement that $M$ is an ideal in $E_{M}$. If $[a, b, c]=0$ whenever $a, b$ or $c$ lies in $M$, then we call $M$ a trivial $T$-module. The one-dimensional trivial $T$-module is usually denoted simply by $k$.

A linear map $\psi: M \rightarrow N$ between $T$-modules is a morphism provided the induced map $E_{M} \rightarrow E_{N}$ is a morphism in $\mathbf{L T S}$. Let $T$-Mod (resp., $T-\mathbf{m o d}$ ) be the category of all (resp., finite-dimensional) $T$-modules.

Suppose that $\theta$ is an involution of a Lie algebra $\mathfrak{g}$, and let $T$ be the -1-eigenspace of $\theta$. A $\mathfrak{g}$-module $V$ is a $(\mathfrak{g}, \theta)$-module provided that $\theta$ acts linearly on $V$ so that 
$\theta(x . v)=\theta(x) \cdot \theta(v)$ for all $x \in \mathfrak{g}, v \in V$. In this case, $\theta$ induces an involution on $\mathfrak{g}_{V}=\mathfrak{g} \oplus V$. The -1 -eigenspace $\mathfrak{g}_{V}^{-}$for this action of $\theta$ is necessarily a Lie triple system. Now, if $M$ denotes the -1-eigenspace of $\theta$ on $V, \mathfrak{g}_{V}^{-}$identifies with $E_{M}=T \oplus M$, hence $M$ is a $T$-module. This process determines an exact additive functor of abelian categories

$$
j^{*}:(\mathfrak{g}, \theta)-\operatorname{Mod} \rightarrow T-\operatorname{Mod}, \quad V \mapsto M .
$$

Now let $T$ be an arbitrary Lie triple system, and consider the pair $\left(L_{u}(T), \theta\right)$ defined in 92 It will be advantageous to work with the algebra $K(T)$ defined at the end of $\$ 2$ Clearly, the categories $K(T)-\operatorname{Mod}$ and $\left(L_{u}(T), \theta\right)-\mathbf{M o d}$ are isomorphic. Let $j^{*}: K(T)-$ Mod $\rightarrow T$-Mod be the functor corresponding to the functor $j^{*}$ defined just above, i.e., $j^{*} V$ is the -1 -eigenspace of $\theta$ on $V$, with its induced $T$ module structure. Observe that if $V \in K(T)$-Mod is trivial, i.e., $V$ is a direct sum of copies of $k^{+}$as defined in $\$ 2$ then $j^{*} V=0$. Conversely, if $j^{*} V=0$, then $\theta$ is the identity operator on $V$; so $T$ and hence $L_{u}(T)$ must annihilate $V$, and $K(T)$ acts on $V$ via the augmentation $\epsilon^{+}$, i.e., $V$ is trivial. On the other hand, the onedimensional $K(T)$-module $k^{-}$, upon which $K(T)$ acts via $\epsilon^{-}$, satisfies $j^{*} k^{-} \cong k$, i.e., $j^{*} k^{-}$is the one-dimensional trivial module for $T$.

Let $M$ be a module for $T$ and form the Lie algebra

$$
L_{s}\left(E_{M}\right)=T \oplus M \oplus \operatorname{InnDer}\left(E_{M}\right) .
$$

Letting $U_{M}$ be the subspace of $\operatorname{InnDer}\left(E_{M}\right)$ spanned by the $D_{a, m}=-D_{m, a}, a \in$ $T, m \in M$, define

$$
j_{*}^{\prime} M:=M \oplus U_{M}=M \oplus[T, M] .
$$

Because $T$ is a Lie triple subsystem of $E_{M}$, the inclusion $T \subset E_{M}$ extends to a Lie algebra morphism $L_{u}(T) \rightarrow L_{s}\left(E_{M}\right)$. Under this map, $j_{*}^{\prime}(M)$ is the $L_{u}(T)$ submodule of $L_{s}\left(E_{M}\right)$ generated by $M$. The involution $\theta \in \operatorname{Aut}\left(L_{u}(T)\right)$ acts compatibly with the corresponding involution on $L_{s}\left(E_{M}\right)$, and the subspace $j_{*}^{\prime} M$ of $L_{s}\left(E_{M}\right)$ is $\theta$-stable. Under the $\theta$-action on $j_{*}^{\prime} M, M$ is exactly the -1-eigenspace. Thus, $j_{*}^{\prime}(M)$ is a $K(T)$-module satisfying $j^{*} j_{*}^{\prime} \cong \mathrm{id}$.

In general, a morphism $T^{\prime} \rightarrow T^{\prime \prime}$ of Lie triple systems need not induce a morphism $L_{s}\left(T^{\prime}\right) \rightarrow L_{s}\left(T^{\prime \prime}\right)$ of Lie algebras. In particular, a morphism $\psi: M \rightarrow N$ of $T$-modules need not induce a morphism $L_{s}\left(E_{M}\right) \rightarrow L_{s}\left(E_{N}\right)$. However, the definitions imply that $\psi$ does give a well-defined linear map $j_{*}^{\prime}(\psi): M \oplus U_{M} \rightarrow N \oplus U_{N}$; in fact, for $a_{i} \in T, m_{i} \in M, \sum D_{a_{i}, m_{i}}=0$ in $U_{M}$ if and only if $\sum\left[a_{i}, m_{i}, t\right]=0$ for all $t \in T$. If this happens, then $\sum D_{a_{i}, \psi\left(m_{i}\right)}=0$ in $U_{N}$. In addition, it is easily verified that $j_{*}^{\prime}(\psi)$ is a morphism of $L_{u}(T)$-modules. Thus, we obtain a functor $j_{*}^{\prime}: T-\operatorname{Mod} \rightarrow K(T)-$ Mod.

Proposition 3.2. The functor $j_{*}^{\prime}: T-\operatorname{Mod} \rightarrow K(T)-\operatorname{Mod}$ satisfies $j^{*} j_{*}^{\prime} \cong \mathrm{id}$. For $0 \neq M \in T$-Mod, $j_{*}^{\prime} M$ has no nonzero trivial submodule. In other words, $\operatorname{Hom}_{K(T)}\left(k^{+}, j_{*}^{\prime} M\right)=0$. The functor $j_{*}^{\prime}$ preserves both surjections and injections.

Proof. The final statement above follows immediately from the definition. It remains only to check the penultimate assertion for a nonzero $T$-module $M$. Suppose that $N$ is a $K(T)$-submodule of $j_{*}^{\prime} M$ satisfying $j^{*} N=0$. Then $N \subseteq U_{M}$, while $T N \subset M$ implies $T N=0$. Suppose $x=\sum D_{a_{i}, m_{i}} \in N, a_{i} \in T, m_{i} \in M$. Then for all $m \in M, t \in T, \sum\left[a_{i}, m_{i}, t\right]=0=\sum\left[a_{i}, m_{i}, m\right]$. Hence, by definition of $U_{M}$, $x=0$, and so $N=0$. 
In [12, $j_{*}^{\prime} M$ is called the standard extension of $M$, by way of analogy with the standard enveloping Lie algebra $L_{s}(T)$. On the other hand, (3.3) below introduces a module-theoretic analogue $j_{!} M$ for the universal enveloping Lie algebra $L_{u}(T)$.

Proposition 3.3. The exact functor $j^{*}: K(T)-\mathbf{M o d} \rightarrow T-\mathbf{M o d}$ has a left adjoint $j_{!}$satisfying $j^{*} j_{!} \cong \mathrm{id}$. The functor $j_{\text {! }}$ is right exact.

Proof. For $M \in T$-Mod, consider the $K(T)$-submodule $j_{!} M$ of $L_{u}\left(E_{M}\right)$ generated by $M$. Any morphism $M \rightarrow N$ in $T$-Mod induces a morphism $L_{u}\left(E_{M}\right) \rightarrow L_{u}\left(E_{N}\right)$ of Lie algebras with involution, hence of $K(T)$-modules, and finally a morphism $j_{!} M \rightarrow j_{!} N$ of $K(T)$-modules. Thus, $j_{\text {! }}$ is a functor which clearly satisfies $j^{*} j_{!} \cong \mathrm{id}$. Given $V \in K(T)$-Mod, any morphism $M \rightarrow j^{*} V$ in $T$-Mod uniquely defines a morphism $E_{M} \rightarrow E_{j^{*} V}$ in $\mathbf{L T S}_{k}$. Since $E_{j^{*} V}$ is a subsystem of $L_{u}(T) \oplus V$, the map $M \rightarrow j^{*} V$ uniquely extends to a morphism $L_{u}\left(E_{M}\right) \rightarrow L_{u}(T) \oplus V$ of Lie algebras which commutes with the natural actions of $\theta$. Hence, we obtain a unique $K(T)$-module morphism $j_{!} M \rightarrow V$ which extends the original morphism $M \rightarrow j^{*} V$. Thus, $j$ ! defines a left adjoint to $j^{*}$. Since $j$ ! is a left adjoint, it is right exact.

Example 3.4. The isomorphism $k \stackrel{\sim}{\rightarrow} j^{*} k^{-}$in $T-$ Mod induces a short exact sequence

$$
0 \rightarrow V \rightarrow j ! k \rightarrow k^{-} \rightarrow 0
$$

in $K(T)$-Mod in which $V$ is necessarily a trivial $K(T)$-module. By adjointness, $\operatorname{Hom}_{K(T)}(j ! k, j ! k) \cong \operatorname{Hom}_{T}\left(k, j^{*} j_{!} k\right)=\operatorname{Hom}_{T}(k, k)=k$; so $j ! k$ is indecomposable.

Next, applying the covariant functor $\operatorname{Hom}_{K(T)}\left(j_{!} k,-\right)$ to any extension

$$
0 \rightarrow k^{+} \rightarrow E \rightarrow j_{!} k \rightarrow 0
$$

would induce an injection $\operatorname{Hom}_{k(T)}(j ! k, E) \rightarrow \operatorname{Hom}_{K(T)}\left(j_{!} k, j_{!} k\right)$, since $\operatorname{Hom}_{K(T)}\left(j ! k, k^{+}\right)=0$. Then since $j^{*} j_{1} E \cong k$ and $j^{*} j_{!} k \cong k, \operatorname{Hom}_{k(T)}\left(j_{!} k, E\right) \cong$ $k \cong \operatorname{Hom}_{K(T)}\left(j ! k, j_{!} k\right)$, whence the injection is an isomorphism. Thus, pulling back the identity map on $j_{!} k$ to $E$ yields a splitting of the extension above.

Since $\operatorname{Ext}_{K(T)}^{1}\left(j ! k, k^{+}\right)$equals zero, the long exact sequence for $\operatorname{Ext}_{K(T)}\left(-, k^{+}\right)$ applied to this short exact sequence implies that

$$
V^{*}=\operatorname{Hom}_{k}(V, k) \cong \operatorname{Hom}_{K(T)}\left(V, k^{+}\right) \cong \operatorname{Ext}_{K(T)}^{1}\left(k^{-}, k^{+}\right) .
$$

In $\S 6$ below, we will see that if $T$ is finite-dimensional, then the cohomology groups $\operatorname{Ext}_{K(T)}^{\bullet}(V, W)$ are finite-dimensional for $V, W \in T-\mathbf{m o d}$.

In what follows, it will be convenient to make use of the elementary theory of quotient categories, as discussed in [6 $\$ 15]$, for example. Let $\mathcal{S}$ be the kernel of $j^{*}: K(T)-$ Mod $\rightarrow T$-Mod, i.e., the full subcategory of $K(T)$-Mod consisting of objects $V$ satisfying $j^{*} V=0$. Since $j^{*}$ is exact, $\mathcal{S}$ is a Serre subcategory of $K(T)$-Mod in the sense that for any short exact sequence $0 \rightarrow A \rightarrow B \rightarrow C \rightarrow 0$ in $K(T)$-Mod, $B \in \mathcal{S}$ if and only if both $A, C \in \mathcal{S}$. We have the following result, which identifies $T$-Mod in terms of $K(T)$-Mod.

Theorem 3.5. Let $T$ be a Lie triple system. Then T-Mod is isomorphic to a quotient category of $K(T)-\mathbf{M o d}$, namely $T-\mathbf{M o d} \cong K(T)-\mathbf{M o d} / \mathcal{S}$.

Proof. By (the dual of) [6, 15.18] applied to the pair $\left(j_{!}, j^{*}\right), T$-Mod is isomorphic to the quotient category $K(T)-\operatorname{Mod} / \mathcal{S}$. 
Corollary 3.6. Assume $T$ is a Lie triple system. Then the category T-Mod has enough injectives. In fact, every object in T-Mod has an injective envelope.

Proof. Let $M \in T$-Mod. Then $j_{*}^{\prime} M \in K(T)$-Mod has no nonzero subobject lying in $\mathcal{S}$ by Proposition 3.2. Thus, if $I$ is an injective envelope of $j_{*}^{\prime} M$ in $K(T)-$ Mod (which always exists), then by [6, 15.22], $j^{*} I$ is an injective envelope of $j^{*} j_{*}^{\prime} M \cong$ $M$.

In the result below, let $\operatorname{Irr}(T)$ (resp., $\operatorname{Irr}(K(T))$ ) denote the "set" of irreducible $T$-modules (resp., $K(T)$-modules). (We identify isomorphic irreducible modules.) Let $\operatorname{Irr}(K(T))^{\prime}$ be the subset of $\operatorname{Irr}(K(T))$ consisting of all nontrivial irreducible modules.

Corollary 3.7. Let $T$ be a Lie triple system. The functor $j^{*}$ maps $\operatorname{Irr}(K(T))^{\prime}$ bijectively onto the set $\operatorname{Irr}(T)$.

Proof. For $L \in \operatorname{Irr}(K(T))^{\prime}, j^{*} L \neq 0$. If $M$ is a nonzero submodule of $j^{*} L$, the inclusion map $M \rightarrow j^{*} L$ implies the existence of a nonzero map $j ! M \rightarrow L$, which must then be surjective since $L$ is irreducible. Now the exactness of $j^{*}$ implies that $M=j^{*} L$, so that $j^{*} L$ is an irreducible $T$-module. Given another $L^{\prime} \in \operatorname{Irr}(K(T))^{\prime}$ such that $j^{*} L^{\prime} \cong j^{*} L$, the definition of morphisms in a quotient category [6. (15.3)] implies that $L \cong L^{\prime}$. Finally, it remains to show that if $S \in \operatorname{Irr}(T)$, then there exists $L \in \operatorname{Irr}(K(T))^{\prime}$ such that $j^{*} L \cong S$. Let $L=j ! S / V$, where $V$ is the sum of all trivial submodules of $j ! S$ (i.e., $V$ is the largest trivial submodule of $j ! S$ ). Then $L$ has no trivial submodules. On the other hand, $\operatorname{Hom}_{K(T)}\left(j ! S, k^{+}\right) \cong \operatorname{Hom}_{T}\left(S, j^{*} k^{+}\right)=0$, so that $j ! S$ and hence $L$ have no trivial quotient modules. It follows immediately that $L \in \operatorname{Irr}(K(T))^{\prime}$ and $j^{*} L \cong S$.

Definition/Remark 3.8. Using the results developed so far, we can place the categories $T$-Mod and $K(T)$-Mod in a diagram which will play an essential role throughout this paper. Let $\mathbf{V e c}_{k}$ be the category of vector spaces over $k$. Let $i_{*}: \mathbf{V e c}_{k} \rightarrow K(T)-\mathbf{M o d}$ be the exact functor that assigns to any vector space the corresponding trivial $K(T)$-module. We then have a diagram

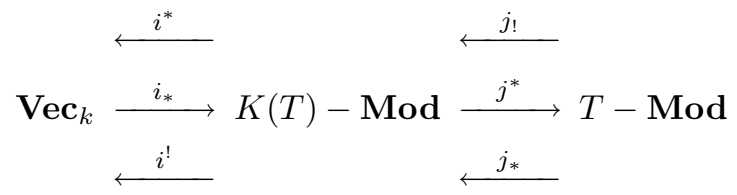

in which $\left(i^{*}, i_{*}, i^{!}\right)$and $\left(j_{!}, j^{*}, j_{*}\right)$ are adjoint triples. Here $i_{*}, j_{!}, j^{*}$ are the functors defined earlier, and the functors $i^{*}, i^{!}$are defined as follows. Given $V \in K(T)-\mathbf{M o d}$, let $i^{*} V$ (resp., $i^{!} V$ ) be the largest quotient (resp., submodule) of $V$ that is $K(T)$ trivial. Observe that $i^{!} V$ is merely the sum of all trivial submodules of $V$. If $\left\{N_{i}\right\}$ is a collection of all submodules of $V$ such that $V / N_{i}$ is trivial, then $i^{!} V \cong V / \cap N_{i}$. The functor $i^{*}$ is a left adjoint to $i_{*}$, while $i^{\text {! }}$ is a right adjoint to $i_{*}$. We now define $j_{*}$. By [6, (15.23), (15.14)] and Corollary [3.6, $j^{*}$ has a right adjoint $j_{*}$ satisfying $j^{*} j_{*} \cong$ id. Notice that $i^{*} j_{!}=0$ and $i^{!} j_{*}=0$. Also, we have $i^{*} i_{*} \cong i^{!} i_{*} \cong$ id and $j^{*} j_{\text {! }} \cong \mathrm{id}$. For any $V \in K(T)-$ Mod, there exist exact sequences

$$
0 \rightarrow i_{*} i^{!} V \rightarrow V \rightarrow j_{*} j^{*} V, \quad j ! j^{*} V \rightarrow V \rightarrow i_{*} i^{*} V \rightarrow 0
$$


We caution the reader that the functor $j_{*}$ defined above is not in general the same as the functor $j_{*}^{\prime}$ defined earlier in this section. The following result establishes a number of important properties of the functor $j_{*}$, as well as giving another description of $j_{*}^{\prime}$ which clarifies the relationship between $j_{*}$ and $j_{*}^{\prime}$.

Theorem 3.9. Suppose $M \in T$-Mod. Then:

(a) $\operatorname{Hom}_{K(T)}\left(k^{+}, j_{*} M\right)=0$. In other words, $j_{*} M$ has no nonzero $K(T)$ submodule $N$ satisfying $j^{*} N=0$.

(b) For any $N \in K(T)-\operatorname{Mod}$ such that $\operatorname{Hom}_{K(T)}\left(k^{+}, N\right)=0$ and $j^{*} N \cong M$, there exists a short exact sequence

$$
0 \rightarrow N \rightarrow j_{*} M \rightarrow i_{*} V \rightarrow 0
$$

for some $V \in \mathbf{V e c}_{k}$. In particular, such a sequence exists taking $N=j_{*}^{\prime} M$.

(c) Let $d=\operatorname{dim}_{\operatorname{Ext}_{K(T)}^{1}}^{1}\left(k^{+}, j_{*}^{\prime} M\right) \in \mathbb{N} \cup\{\infty\}$. Then letting $V$ be as in (b) with $N=j_{*}^{\prime} M$, we have $\operatorname{dim} V=d$. Also, $\operatorname{Ext}_{K(T)}^{1}\left(k^{+}, j_{*} M\right)=0$. Thus, $j_{*} M$ may be characterized as the "maximal" extension of $j_{*}^{\prime} M$ by a trivial $K(T)$-module.

(d) For $n \geq 0$, let $R^{n} j_{*}: T-\mathbf{M o d} \rightarrow K(T)-\mathbf{M o d}$ be the $n^{\text {th }}$ right derived functor of $j_{*}$. Then for $n>0, j^{*} R^{n} j_{*}=0$, i.e., $R^{n} j_{*}$ takes values in $i_{*} \mathbf{V e c}_{k}$.

(e) We have $i^{*} j_{*}^{\prime} M=0$. Thus, $j_{*}^{\prime} M$ is the kernel of the adjunction morphism $j_{*} M \rightarrow i_{*} i^{*} j_{*} M$.

Proof. Since $j^{*} k^{+}=0$, part (a) follows from adjoint associativity. To see (b), let $N$ satisfy the stated conditions. Then, from

$$
\operatorname{Hom}_{K(T)}\left(N, j_{*} M\right) \cong \operatorname{Hom}_{T}\left(j^{*} N, M\right) \cong \operatorname{Hom}_{T}(M, M),
$$

there exists a morphism $f: N \rightarrow j_{*} M$ such that $j^{*}(f)=$ id. Letting $C=\operatorname{Ker}(f)$ and $D=\operatorname{Coker}(f)$, we form the exact sequence $0 \rightarrow C \rightarrow N \stackrel{f}{\rightarrow} j_{*} M \rightarrow D \rightarrow 0$. Since $j^{*}$ is exact, $j^{*} C=j^{*} D=0$; so $C, D \in i_{*} \mathbf{V e c}_{k}$. Thus, $C=0$ by hypothesis on $N$, and $D=i_{*} V$ for some $V \in \mathbf{V e c}_{k}$. This proves the first assertion in (b) and the second follows from Proposition 3.2

We next prove (c). First, observe that $\operatorname{Ext}_{K(T)}^{1}\left(k^{+}, k^{+}\right)=0$, since $\mathcal{S}=\operatorname{Ker}\left(j^{*}\right)$ is a Serre subcategory of $K(T)-\mathbf{M o d}$ and $\mathcal{S} \cong \mathbf{V e c}_{k}$. Now, suppose that $N \in$ $K(T)$-Mod satisfies

$$
\operatorname{Hom}_{K(T)}\left(k^{+}, N\right)=0 \neq \operatorname{Ext}_{K(T)}^{1}\left(k^{+}, N\right) \text {. }
$$

Then there exists a nonsplit extension $0 \rightarrow N \rightarrow \widehat{N} \rightarrow k^{+} \rightarrow 0$. The long exact sequence of $\operatorname{Ext}_{K(T)}^{\bullet}\left(k^{+},-\right)$gives an exact sequence

$$
\begin{aligned}
0=\operatorname{Hom}_{K(T)}\left(k^{+}, N\right) & \rightarrow \operatorname{Hom}_{K(T)}\left(k^{+}, \widehat{N}\right) \\
& \rightarrow \operatorname{Hom}_{K(T)}\left(k^{+}, k^{+}\right) \rightarrow \operatorname{Ext}_{K(T)}^{1}\left(k^{+}, N\right) \\
& \rightarrow \operatorname{Ext}_{K(T)}^{1}\left(k^{+}, \widehat{N}\right) \rightarrow \operatorname{Ext}_{K(T)}^{1}\left(k^{+}, k^{+}\right)=0 .
\end{aligned}
$$

Since the extension is nonsplit, the map $\operatorname{Hom}_{K(T)}\left(k^{+}, \widehat{N}\right) \rightarrow \operatorname{Hom}_{K(T)}\left(k^{+}, k^{+}\right)$ must be the zero map; so $\operatorname{Hom}_{K(T)}\left(k^{+}, \widehat{N}\right)=0$. Thus, $\operatorname{dim}_{\operatorname{Ext}_{K(T)}^{1}}^{1}\left(k^{+}, N\right)=$ $\operatorname{dim} \operatorname{Ext}_{K(T)}^{1}\left(k^{+}, \widehat{N}\right)+1$ (where we define $\left.\infty+1=\infty\right)$. Observe that $j^{*} N \cong j^{*} \widehat{N}$.

Thus, if $d=\infty$, we can repeatedly apply this process, beginning with $N=j_{*}^{\prime} M$, to obtain for any positive integer $n$ an extension $0 \rightarrow j_{*}^{\prime} M \rightarrow B_{n} \rightarrow i_{*} V_{n} \rightarrow 0$ with $\operatorname{Hom}_{K(T)}\left(k^{+}, B_{n}\right)=0, \operatorname{dim} V_{n}=n$, and $j^{*} B_{n} \cong M$. By (b), $B_{n} \subset j_{*} M$, so that $\operatorname{dim} V=\infty$. If $d<\infty$, we have that $\operatorname{dim} V \geq d$ and $\operatorname{Ext}_{K(T)}^{1}\left(k^{+}, B_{d}\right)=0$. By (b), 
we have a short exact sequence $0 \rightarrow B_{d} \rightarrow j_{*} M \rightarrow i_{*} W \rightarrow 0$ for some vector space $W$. If $W \neq 0$, the pullback of this sequence through an inclusion $k^{+} \subseteq W$ gives an exact sequence $0 \rightarrow B_{d} \rightarrow \widetilde{B} \rightarrow k^{+} \rightarrow 0$, where $\widetilde{B} \subseteq j_{*} M$. Since $B_{d}$ has no nonsplit trivial extension, this sequence must split, i.e., there is a nonzero homomorphism $k^{+} \rightarrow \widetilde{B} \subseteq j_{*} M$, which is impossible. Thus, $W=0$ and $B_{d} \cong j_{*} M$, as required.

Therefore, to complete the proof of (c), we must check that $\operatorname{Ext}_{K(T)}^{1}\left(k^{+}, j_{*} M\right)=$ 0 if $d=\infty$. The following argument is independent of $d$ : In $T$-Mod, choose an exact sequence $0 \rightarrow M \rightarrow I \rightarrow C \rightarrow 0$, with $I$ injective. Since $j_{*}$ is left exact, we obtain an exact sequence $0 \rightarrow j_{*} M \rightarrow j_{*} I \rightarrow j_{*} C$ and thus an exact sequence $0 \rightarrow j_{*} M \rightarrow j_{*} I \rightarrow D \rightarrow 0$, where $D \subseteq j_{*} C$. In particular, Hom $K(T)\left(k^{+}, D\right)=0$. Now the long exact sequence for $\operatorname{Ext}_{K(T)}\left(k^{+},-\right)$gives an exact sequence

$$
0 \rightarrow \operatorname{Ext}_{K(T)}^{1}\left(k^{+}, j_{*} M\right) \rightarrow \operatorname{Ext}_{K(T)}^{1}\left(k^{+}, j_{*} I\right) .
$$

Since $j^{*}$ is exact, $j_{*} I$ is injective in $K(T)-\mathbf{M o d}$; so

$$
\operatorname{Ext}_{K(T)}^{1}\left(k^{+}, j_{*} M\right) \cong \operatorname{Ext}_{K(T)}^{1}\left(k^{+}, j_{*} I\right)=0,
$$

completing the proof of (c).

Next, the exactness of $j^{*}$ implies that $j^{*} R^{n} j_{*}=R^{n} j^{*} j_{*} \cong R^{n}$ id $=0$ for $n>0$. This proves $(\mathrm{d})$.

Finally, to see (e), observe that $j_{*}^{\prime} M \in K(T)-$ Mod is generated by $M$, which lies in the -1 -eigenspace of $\theta$. Hence $i^{*} j_{*}^{\prime} M=0$. The remaining assertion follows from (b) with $N=j_{*}^{\prime} M$.

Remark 3.10. If $\operatorname{dim} T<\infty$ in Theorem 3.9 then the hypothesis that $d<\infty$ is always true provided that $\operatorname{dim} M<\infty$. This will be clear from the results below in $₫ 6.2$.

Theorem 3.5 provides one realization of $T$-Mod, but there are several more, which we shall illustrate. In the algebra $K(T)$, form the idempotents

$$
e=\frac{1-\theta}{2}, \quad f=\frac{1+\theta}{2}
$$

which satisfy $1=e+f$.

Recall the relationship relating $K(T) / K(T) e K(T)$-Mod to the module category for the centralizer algebra $e K(T) e \cong \operatorname{End}_{K(T)}(e K(T))$. There is a diagram

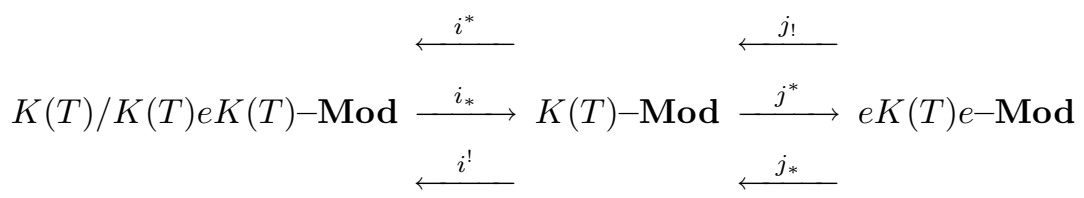

where $\left(i^{*}, i_{*}, i^{!}\right)$and $\left(j_{!}, j^{*}, j_{*}\right)$ are adjoint triples as follows. For $N \in K(T)-\mathbf{M o d}$ and $M \in e K(T) e-$ Mod, set $j^{*} N=e N$, while $j_{*} M=\operatorname{Hom}_{e K(T) e}(e K(T), M)$, and $j_{!} M=K(T) e \otimes_{e K(T) e} M$. If $N \in K(T)-\operatorname{Mod}$, let $i^{*} N$ be the largest quotient of $N$ that has a $K(T) / K(T) e K(T)$-module structure. Let $i^{!} N$ be the largest submodule of $N$ that is annihilated by $K(T) e K(T)$. For $M \in K(T) / K(T) e K(T)-$ Mod, take $i_{*} M$ to be $M$ regarded as a $K(T)$-module via the canonical projection.

The image under $i_{*}$ of $K(T) / K(T) e K(T)$-Mod identifies with the kernel of $j^{*}$ (which is a Serre subcategory of $K(T)-\mathbf{M o d}$ ), and the category $e K(T) e-\mathbf{M o d}$ is isomorphic to the quotient category $K(T) / K(T) e K(T)-$ Mod. The following result 
shows that our choice of notation $\left(i^{*}, i_{*}, i^{!}, j_{!}, j^{*}, j_{*}\right)$ does not conflict with that in Definition/Remark 3.8.

Theorem 3.13. Let $T$ be a Lie triple system and let $e \in K(T)$ be as in (3.11). Then $T-\mathbf{M o d} \cong e K(T) e-$ Mod. Furthermore, the functors in Definition/Remark 3.8 correspond to those in (3.12).

Proof. Let $N \in K(T)$-Mod. By construction, the ideal $K(T) e K(T)$ annihilates $N \in K(T)$-Mod exactly when $N$ has zero -1-eigenspace under the action of $\theta$, i.e., when $j^{*} N=0$. Thus, we have $(K(T) / K(T) e K(T))-\mathbf{M o d} \cong \mathcal{S} \cong \mathbf{V e c}_{k}$ and $e K(T) e-\mathbf{M o d} \cong T-\mathbf{M o d}$ by Theorem 3.5. The other statements are now clear.

Although Corollary 3.6 implies that $T-$ Mod has enough injectives, Theorem 3.13 provides an alternate means of drawing this conclusion, along with additional useful information about $T$-Mod.

Corollary 3.14. Assume that $T$ is a Lie triple system. Then T-Mod has enough projectives and enough injectives.

The following result also follows from Theorem 3.13 but can be checked directly.

Lemma 3.15. If $J=\operatorname{Ker}\left(\epsilon^{+}\right)$is the augmentation ideal of $K(T)$, then $K(T) e K(T)$ $=J$.

Proof. Since $\theta e=e \theta=-e, K(T) e K(T)=U(T) e U(T)$. So it suffices to verify that $J$ equals the ideal $U(T) e U(T)$. Because $\epsilon^{+}(e)=0, U(T) e U(T) \subseteq J$. Since $\epsilon^{+}(f)=1, J=U(T)_{+} f \oplus U(T) e$, where $U(T)_{+}=\operatorname{Ker}(\epsilon)$ is the augmentation ideal of $U(T)$. Hence it suffices to show that $U(T)_{+} \subset U(T) e U(T)$. If $a \in U(T)$ satisfies $\theta(a)=-a$, then $a=e a+a e \in U(T) e U(T)$. Hence $T \subset U(T) e U(T)$. Since $L_{u}(T)=T+[T, T], T$ generates $U(T)_{+}$and so $U(T)_{+} \subset U(T) e U(T)$, as required.

As a consequence of Lemma 3.15, $K(T) / K(T) e K(T) \cong k$, as expected.

There is yet another way to approach the category $T$-Mod. For any $a+b \theta \in$ $K(T), \theta e=-e$ implies $e(a+b \theta) e=e(a-b) e$, with $a-b \in U(T)$. For $a \in U(T)$, write $a=a_{+}+a_{-}$, where $\theta\left(a_{ \pm}\right)= \pm a_{ \pm}$. Then

$$
e a e=e a_{+} e=e a_{+}=a_{+} e .
$$

As a consequence, $e K(T) e=\{a e \mid a \in U(T)$ and $\theta(a)=a\}$. Thus, if $U(T)^{\theta}=$ $\{a \in U(T) \mid \theta(a)=a\}$, then $U(T)^{\theta} \rightarrow e K(T) e, a \mapsto e a e=e a=a e$ is an algebra isomorphism. Applying Theorem 3.13 we obtain the following explicit description of $T$-Mod.

Corollary 3.16. If $T$ is a Lie triple system, then $T-\operatorname{Mod} \cong U(T)^{\theta}-\mathbf{M o d}$.

Remarks 3.17. (a) Suppose $T=\mathfrak{p}$ as in Example 2.4. Then in all but a few cases, $\mathfrak{g} \cong L_{u}(\mathfrak{p})$, and hence $\mathfrak{p}-\operatorname{Mod} \cong U(\mathfrak{g})^{\theta}-$ Mod.

(b) Let $\mathfrak{k}=[T, T]$. Since $T-\mathbf{M o d} \cong U(T)^{\theta}-\mathbf{M o d}$, the natural inclusion $U(\mathfrak{k}) \subseteq$ $U(T)^{\theta}$ provides an exact restriction functor $r: T-\mathbf{M o d} \rightarrow \mathfrak{k}$-Mod. Also, suppose that $x_{1}, \ldots, x_{m}$ is a basis for $T$. Let $V$ be the subspace of $U(T)^{\theta}$ spanned by monomials $x_{1}^{a_{1}} \cdots x_{m}^{a_{m}}$ with $a_{1}+\cdots+a_{m}$ even. Then $V$ is a free basis for $U(T)^{\theta}$ as either a right or a left $U(\mathfrak{k})$-module. 
(c) In general, the functor $j_{*}: T-\mathbf{M o d} \rightarrow K(T)-$ Mod is not exact; in fact, it can happen that the right derived functors $R^{m} j_{*}$ do not vanish for infinitely many values of the nonnegative integer $m$. For example, consider the situation in Example 2.5 in which $\operatorname{dim}(T)=2$. In this case, $L_{u}(T)$ has a basis $x, y, z$, with $[x, y]=z$ and $z$ central. The functor $j_{*}$ identifies with the left exact functor $\operatorname{Hom}_{U(T)^{\theta}}(U(T),-)$; so, for $m>0$,

$$
R^{m} j_{*}(-)=\operatorname{Ext}_{U(T)^{\theta}}^{m}(U(T),-) .
$$

Both $U(T)$ and $U(T)^{\theta}$ are free modules over the central subalgebra $Z=k[z] \subset$ $U(T)^{\theta}$. Thus, if $U(T)$ has a finite projective $U(T)^{\theta}$-resolution $P_{\bullet} \rightarrow U(T) \rightarrow 0$, we can apply the functor $-\otimes_{Z} k=-\otimes_{Z} Z / z Z$ to obtain that the polynomial ring $R=k[x, y] \cong U(T) \otimes_{Z} k$ has finite projective dimension over the subring $S=k\left[x^{2}, x y, y^{2}\right] \cong U(T)^{\theta} \otimes_{z} k$. Let $R^{\prime}$ be the $S$-submodule of $R$ spanned by the odd monomials $x^{i} y^{j}$ (i.e., with $i+j$ odd). There is a short exact sequence

$$
0 \rightarrow R^{\prime} \stackrel{\eta}{\rightarrow} S \oplus S \stackrel{\epsilon}{\rightarrow} R^{\prime} \rightarrow 0
$$

in which $\eta(f)=(y f,-x f)$ and $\epsilon(f, g)=x f+y g$. These splice together to define a projective resolution $Q_{\bullet} \stackrel{\epsilon}{\rightarrow} R^{\prime} \rightarrow 0$ in which $Q_{i}=S \oplus S$, while $Q_{i} \rightarrow Q_{i-1}$ is defined by $(f, g) \mapsto\left(x y f+y^{2} g,-x^{2} f-x y g\right)$. Since the differentials in the

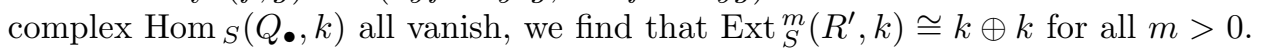
Because $R=S \oplus R^{\prime}$, we conclude that $R$ does not have finite projective dimension as an $S$-module, a contradiction. Thus, $T$-Mod does not have finite homological dimension.

Our final comments in this section address the issue of alternative notions of a module for a Lie triple system $T$.

Remarks 3.18. (a) The first alternative notion we discuss comes from [19], and is suggested by the view of a module for a Lie algebra $\mathfrak{g}$ as a vector space $V$ with an action determined by a Lie algebra morphism $\mathfrak{g} \rightarrow \mathfrak{g l}(V)$. Namely, call a vector space $V$ a $T$-module provided that there is given a morphism $\rho: T \rightarrow \mathfrak{g l}(V)_{\text {trip }}$ of Lie triple systems. Now, by the universal property of $L_{u}(T), \rho$ extends uniquely to a Lie algebra morphism $\widetilde{\rho}: L_{u}(T) \rightarrow \mathfrak{g l}(V)$. Conversely, every $L_{u}(T)$-module $V$ is determined by a Lie algebra homomorphism $L_{u}(T) \rightarrow \mathfrak{g l}(V)$ which yields a Lie triple system morphism $T \rightarrow \mathfrak{g l}(V)_{\text {trip. }}$. Thus, the category of " $T$-modules" in this sense identifies with the category of $L_{u}(T)$-Mod, and so leads to nothing new from the point of view of representation theory.

(b) Let $V$ be a (finite-dimensional) vector space. In [30], a representation of $T$ (resp., a weak representation of $T$ ) is defined in terms of two bilinear mappings $E, \psi: T \times T \rightarrow$ End $(V)$. Let $a, b, c, d \in T$, and consider the following relations on $E$ and $\psi$ :

(i) $E(a, b)=\psi(b, a)-\psi(a, b)$;

(ii) $[E(a, b), \psi(c, d)]=\psi([a b c], d)+\psi(c,[a b d])$;

(iii) $\psi(c, d) \psi(a, b)-\psi(b, d) \psi(a, c)-\psi(a,[b c d])+E(b, c) \psi(a, d)=0$,

where $[E(a, b), \psi(c, d)]$ is the usual commutator bracket on $\mathfrak{g l}(V)$. The mappings $E, \psi$ give the vector space $V$ the structure of a weak $T$-module if they satisfy (i) and (ii). If, in addition, $E$ and $\psi$ also satisfy (iii), then $V$ is called a $T$-module; this yields the same notion previously defined in [29].

Set $E(a, b)$ to be the inner derivation $D_{a, b}$ of $T$, and put $\psi(a, b):=\tilde{D}_{a, b}$ defined by $\tilde{D}_{a, b}(x)=[x a b]$ for $x \in T$. Then $T$ itself becomes a $T$-module. In fact, one can 
check that any vector space, closed under a trilinear ternary product for which the maps $D_{a, b}$ and $\tilde{D}_{a, b}$ above satisfy conditions (i) and (ii), is necessarily a Lie triple system. As a consequence, any $M \in T$-Mod becomes a weak $T$-module by setting $\psi(a, b)(m)=[m a b]$ and $E(a, b)(m)=[a b m]$; moreover, $M$ is also a $T$-module in the sense of [29] or [30]. However, such a $T$-module need not be an element of $T$-Mod. In terms of the cohomology $H_{Y}^{n}(T, M)$, defined for such modules in [29], a $T$-module would yield an object of $T$-Mod in our sense only if $H_{Y}^{3}(T, M)=0$ 29, Cor. to Thm. 2, p. 50]. It is also worth pointing out that if $\rho: T \rightarrow \mathfrak{g l}(V)_{\operatorname{trip}}$ defines a $T$-module as in (a), then $V$ becomes a module in the sense of [29], [30], upon setting $\psi(a, b)=\rho(a) \rho(b)$.

(c) This remark is based on discussions between the first author and Michael Kinyon. Given a category $\mathcal{C}$ with products, let $T \in \mathcal{C}$ be a fixed object. The notion of the "category of $T$-modules" can be formulated as follows: let $\mathcal{C} / T$ be the category having as objects the morphisms $E \rightarrow T$ in $\mathcal{C}$. The morphisms in $\mathcal{C} / T$ are defined in the evident way (i.e., $\mathcal{C} / T$ is the comma category over $X[21$, p. $46])$. Let $T-\mathbf{M o d}^{\prime}$ be the subcategory of $\mathcal{C} / T$ consisting of "vector space objects" $E \rightarrow T$ over $k$. The idea of taking $T-\mathbf{M o d}^{\prime}$ as the category of $T$-modules has been attributed to Eilenberg; cf. [24, p. 5.15], but see especially [26, Ch. III] for a detailed discussion. In particular, let $\mathcal{C}=\mathbf{L T S}_{k}$ be the category of Lie triple systems over $k$. Let $\pi: E \rightarrow T$ be an object in $T$-Mod'. The "zero object" in $T-$ Mod' $^{\prime}$ is defined by a morphism $0: T \rightarrow E$ splitting $\pi$, so that we can write $E=T \oplus M$, where $M=\operatorname{Ker}(\pi)$. The "addition" on $E \rightarrow T$ is defined by a morphism $+: E \times_{T} E \rightarrow E$ in $\mathcal{C} / T$. A somewhat lengthy calculation (left to the interested reader) directly shows that this condition forces $M$ to be an object in $T$-Mod. Conversely, for $M \in T$-Mod, the projection $E_{M} \rightarrow T$ defines an object in $T$-Mod'. It follows that the categories $T-\mathbf{M o d}$ and $T-\mathbf{M o d}^{\prime}$ are equivalent, so that, in some categorical sense, $T$-Mod is the "correct" choice for a category of $T$-modules.

\section{Restricted Lie triple systems}

Throughout this section, the field $k$ has positive characteristic $p>2$. Restricted Lie triple systems were first defined in [14, and we begin by refining the approach taken there. Recall that the definition of a restricted Lie algebra captures features displayed by Lie algebras of derivations, in which the $[p]$-operator acts simply by taking the $p$ th power. The following result due to Jacobson (see, e.g., 17, Ch. V, Thm. 11]) gives an explicit way in which restricted Lie algebras arise.

Theorem 4.1. Let $\mathfrak{g}$ be a Lie algebra over $k$. Suppose that $\mathfrak{g}$ has a basis $\left\{u_{i}\right\}_{i \in I}$ such that, for each $i \in I$, there exists $v_{i} \in \mathfrak{g}$ satisfying $\left(\operatorname{ad} u_{i}\right)^{p}=\operatorname{ad} v_{i}$. Then there exists a unique mapping $(-)^{[p]}: \mathfrak{g} \rightarrow \mathfrak{g}, x \mapsto x^{[p]}$, such that $\left(\mathfrak{g},(-)^{[p]}\right)$ is a restricted Lie algebra and $u_{i}^{[p]}=v_{i}$ for all $i \in I$.

As we shall see, our definition of a restricted Lie triple system will ensure that a similar result holds. This definition requires some new pieces of notation.

Let $\mathfrak{g}$ be any Lie algebra. For any positive integer $n \geq 2$ and elements $x_{1}, x_{2}, \ldots$, $x_{n}$ of $\mathfrak{g}$, set

$$
\left(x_{1}, x_{2}, \ldots, x_{n}\right):=\left[\left[\cdots\left[\left[x_{1}, x_{2}\right], x_{3}\right], \cdots\right], x_{n}\right] \in \mathfrak{g} .
$$


Now let $T$ be any Lie triple system and $N \geq 3$ any positive odd integer. For elements $x_{1}, x_{2}, \ldots, x_{N}$ of $T$, upon taking the triple product, define

$$
\left(x_{1}, x_{2}, \ldots, x_{N}\right):=\left[\left[\cdots\left[\left[x_{1}, x_{2}, x_{3}\right], x_{4}, x_{5}\right], \cdots\right], x_{N-1}, x_{N}\right] \in T .
$$

If $(\mathfrak{g}, \widetilde{T}, \phi) \in \mathbf{L I E}_{k}(T)$, then the expressions (4.2) and (4.3) are equal for any odd $n=N \geq 3$ and any $x_{1}, x_{2}, \ldots, x_{N} \in \widetilde{T}$; so there is no ambiguity in notation.

For $a, b \in T$ and $\lambda$ indeterminate, the expression $(a, \lambda a+b, \lambda a+b, \ldots, \lambda a+b)$, with $\lambda a+b$ occurring $p-1$ times, is a polynomial in $\lambda$ with coefficients in $T$. For $i=1, \ldots, p$, we define $s_{i}(a, b) \in T$ by requiring $i s_{i}(a, b)$ to be the coefficient of $\lambda^{i-1}$ in $(a, \lambda a+b, \lambda a+b, \ldots, \lambda a+b)$. For $(\mathfrak{g}, \widetilde{T}, \phi) \in \mathbf{L I E}_{k}(T)$ and $a, b \in \widetilde{T}$ we have

$$
\begin{aligned}
(a, \lambda a+b, \lambda a+b, \ldots, \lambda a+b) & =(-1)^{p-1}[\lambda a+b,[\lambda a+b,[\cdots,[\lambda a+b, a] \cdots]]] \\
& =(\operatorname{ad}(\lambda a+b))^{p-1}(a) .
\end{aligned}
$$

In this case, $i s_{i}(a, b)$ is thus the coefficient of $\lambda^{i-1}$ in the expression $(\operatorname{ad}(\lambda a+$ $b))^{p-1}(a)$, which agrees precisely with the use of the notation $s_{i}(a, b)$ as in 14 and 17.

Definition 4.4. A restricted Lie triple system is a pair $\left(T,(-)^{[p]}\right)$, where $T$ is a Lie triple system over $k$ together with a mapping $(-)^{[p]}: T \rightarrow T$ such that for all $a, b, c \in T$ and all $\alpha \in k$, the following properties are satisfied:

$$
\begin{aligned}
(\alpha a)^{[p]} & =\alpha^{p} a^{[p]}, \\
(a+b)^{[p]} & =a^{[p]}+b^{[p]}+\sum_{i=1}^{p-1} s_{i}(a, b), \\
{\left[a, b^{[p]}, c\right] } & =(a, b, \ldots, b, c)(p \text { copies of } b) .
\end{aligned}
$$

Together with the identity $[b, a, c]=-[a, b, c]$ for any $a, b, c \in T$, (4.4c) determines any term of the form $\left[a^{[p]}, b, c\right]$. In practice, we suppress mention of $(-)^{[p]}$ and just refer to $T$ as a restricted Lie triple system.

As a basic example, let $G, \theta, \mathfrak{p}$ be as in Example 2.4 Because $\theta$ induces an involution of $\mathfrak{g}$ as a restricted Lie algebra, $\theta\left(x^{[p]}\right)=\theta(x)^{[p]}$ for $x \in \mathfrak{g}$. Thus, if $x \in \mathfrak{p}, x^{[p]} \in \mathfrak{p}$. The axioms for a restricted Lie algebra immediately imply that $\mathfrak{p}$ is a restricted Lie triple system.

The result below shows that the conditions in Definition 4.4 ensure that for each $a \in T \subseteq L_{s}(T),(\operatorname{ad} a)^{p} \in \operatorname{Der}\left(L_{s}(T)\right)$ is inner.

Proposition 4.5. Let $T$ be a restricted Lie triple system. For each $a \in T \subset L_{s}(T)$, ad $a \in \operatorname{Der}\left(L_{s}(T)\right)$ satisfies

$$
(\operatorname{ad} a)^{p}=\operatorname{ad} a^{[p]} .
$$

Proof. Since $T$ generates $L_{s}(T)$, it suffices to show that the derivations $(\operatorname{ad} a)^{p}$ and $\operatorname{ad} a^{[p]}$ agree on $T$. Let $b \in T$. Since $p$ is odd, $(\operatorname{ad} a)^{p}(b)=[a,[\cdots[a, b] \cdots]]=$ $(-1)^{p}(b, a, \ldots, a)=-(b, a, \ldots, a) \in[T, T]$, while on the other hand, ad $a^{[p]}(b)=$ $-\left[b, a^{[p]}\right] \in[T, T]$. The equality $(\operatorname{ad} a)^{p}(b)=\operatorname{ad} a^{[p]}(b)$ now follows from (4.4c), for by construction of $L_{s}(T)$, for any $a, b \in T,\left[a, b^{[p]}, c\right]=(a, b, \ldots, b, c)$ for all $c \in T$ if and only if $\left[a, b^{[p]}\right]=(a, b, \ldots, b)$. 
By applying $(\operatorname{ad} c)^{p}=\operatorname{ad} c^{[p]}$ to $[a, b] \in[T, T]$, we obtain in $T$ the equality

$$
\left[a, b, c^{[p]}\right]=(a, b, c, \ldots, c)(p \text { copies of } c)
$$

for any $a, b, c \in T, 4$

For $T$ restricted, define $\mathbf{L I E}_{k}^{[p]}(T)$ to be the subcategory of $\mathbf{L I E}_{k}(T)$ with objects those triples $(\mathfrak{g}, \tilde{T}, \phi) \in \mathbf{L I E}_{k}(T)$ for which

$$
(\operatorname{ad} \phi(a))^{p}=\operatorname{ad} \phi\left(a^{[p]}\right)
$$

for all $a \in T$. By Proposition $4.5\left(L_{s}(T), T\right.$, id $)$ is an object in $\mathbf{L I E}_{k}^{[p]}(T)$, and, as in the case of $\mathbf{L I E}_{k}(T)$, it is a terminal object.

We now introduce a Lie algebra which will serve as an initial object in $\mathbf{L I E}_{k}^{[p]}(T)$.

Definition 4.8. Let $T$ be a restricted Lie triple system. Set $L_{u}^{[p]}(T)$ to be the quotient of $L_{u}(T)$ by the ideal $\mathcal{I}$ generated by all terms of the form $\left[a^{[p]}, b\right]-$ $(\operatorname{ad} a)^{p}(b), a, b \in T$ (equivalently, by all terms of the form $\left[b, a^{[p]}\right]-(b, a, \ldots, a), p$ copies of $a$ ).

Using Definition 4.4, if $x \in T,[x, \mathcal{I}]=0$; therefore, $\mathcal{I}$ is precisely the subspace spanned by the elements $\left[a, b^{[p]}\right]-(a, b, \ldots, b), a, b \in T \subset L_{u}(T)$ and $\mathcal{I}$ lies in the intersection of the center of $L_{u}(T)$ and $[T, T]$. In particular, $L_{u}^{[p]}(T) \in \mathbf{L I E}_{k}^{[p]}(T)$. We will continue to write $\theta$ for the induced involution of $L_{u}^{[p]}(T)$. It follows that $\left(L_{u}^{[p]}(T), T\right.$, id $)$ is an initial object in $\mathbf{L I E}_{k}^{[p]}(T)$.

Example 4.9. (a) Let $V$ be a finite-dimensional vector space over $k$. By a $p$ operator on $V$ we mean a map $v \mapsto v^{[p]}$ of $V$ to itself satisfying $(\alpha v)^{[p]}=\alpha^{p} v^{[p]}$ and $(u+v)^{[p]}=u^{[p]}+v^{[p]}$ for all $u, v \in V, \alpha \in k$. For any positive integer $n, V^{[p]^{n}}$ is then a subspace of $V$. Since $V^{[p]} \supseteq V^{[p]^{2}} \supseteq V^{[p]^{3}} \supseteq \cdots$, for some positive integer $n$ we have $V_{1}:=V^{[p]^{n}}=V^{[p]^{n+1}}=\cdots$, and there is a "Fitting decomposition" $V=V_{0} \oplus V_{1}$. Here, $V_{0}$ and $V_{1}$ are $(-)^{[p]}$-stable subspaces of $V$, with $(-)^{[p]}$ nilpotent on $V_{0}$, and injective on $V_{1}$ [17, Ex. 19, p. 196]. Thus, by 17, Thm. 13, p. 192], $V_{1}$ has a basis $v_{1}, \ldots, v_{m}$ satisfying $v_{i}^{[p]}=v_{i}, 1 \leq i \leq m$. For more discussion and references on the classification of $p$-operators, see [17, $\mathrm{p}$. 192]; we will not go into this in further detail. The closely related problem of the classification of commutative, unipotent group schemes is discussed in great detail in [5, Ch. 5].

Now suppose that $T$ is an abelian Lie triple system over $k$, which we assume to be finite-dimensional. Then $\left(T,(-)^{[p]}\right)$ is a restricted Lie triple system precisely when $(-)^{[p]}$ is a $p$-operator in the above sense. Furthermore, $T=T_{0} \oplus T_{1}$, where $T_{0}$, $T_{1}$ are restricted subsystems of $T$ such that $(-)^{[p]}$ is nilpotent on $T_{0}$ and injective on $T_{1}$. A restricted Lie triple system $T$ (abelian or not) such that $(-)^{[p]}$ is nilpotent will be called a $[p]$-unipotent Lie triple system; this terminology is consistent with that in $\left[8\right.$. Thus, in the abelian case, $T_{0}$ is a restricted, $[p]$-unipotent, abelian Lie triple system. An abelian, finite-dimensional restricted Lie triple system $T$ in which

\footnotetext{
${ }^{4}$ Equality [4.6 was included in [14] as an additional defining condition of a restricted Lie triple system, where a restricted Lie triple system was defined by using the imbedding of $T$ into $L_{s}(T)$. The first author would like to thank the referee of [14] for pointing out the redundancy of assuming (4.6) in that case.
} 
$(-)^{[p]}$ is injective will be called a toral restricted Lie triple system 5 Thus, $T_{1}$ is a toral restricted subsystem of $T$.

Finally, if $T$ is abelian and $W$ denotes the subspace $T^{[p]}$, then $L_{u}^{[p]}(T) \cong T \oplus$ $\bigwedge^{2} T / W \wedge T$.

(b) Take $T=\mathfrak{p}$ arising from a simple, simply connected algebraic group $G$ with involution $\theta$ as in (2.4). By [14 Thm. 5.2.4], $L_{u}(\mathfrak{p})$ is a restricted Lie algebra. So by Corollary 4.12 below, $\mathfrak{p}$ inherits a restricted Lie triple system structure from $L_{u}(\mathfrak{p})$. The ideal $\mathcal{I}$ of $L_{u}(\mathfrak{p})$ is identically zero; so $L_{u}^{[p]}(\mathfrak{p}) \cong L_{u}(\mathfrak{p})$.

(c) Let $G$ be as in (b), but assume that $L_{u}(\mathfrak{p})=L_{s}(\mathfrak{p})=\mathfrak{g}$; see Example 2.4. Let $\mathfrak{s}$ be a restricted Lie triple subsystem of $\mathfrak{p}$ and assume that $\mathfrak{s}$ is toral as defined in (a). Then $\mathfrak{s}$ has a basis $\left\{x_{1}, \ldots, x_{m}\right\}$ consisting of elements $x_{i}$ satisfying $x_{i}^{[p]}=x_{i}$. For $1 \leq i, j \leq m$, we have

$$
\left[x_{i}, x_{j}\right]=\left[x_{i}, x_{j}^{[p]}\right]=\left(x_{i}, x_{j}, \ldots, x_{j}\right)=0,
$$

since $\left[x_{i}, x_{j}, x_{j}\right]=0$. Thus, $\mathfrak{s}$ is an abelian Lie subalgebra of $\mathfrak{g}$.

A torus $S \subseteq G$ is called anisotropic provided that $\theta(s)=s^{-1}$ for all $s \in S$. If $S$ is anisotropic, then $\mathfrak{s}=\operatorname{Lie}(S) \subseteq \mathfrak{p}$ is a restricted toral subsystem of $\mathfrak{p}$ which is also a restricted abelian subalgebra of $\mathfrak{g}$. Conversely, let $\mathfrak{s} \subseteq \mathfrak{p}$ be a maximal restricted toral subsystem of $\mathfrak{p}$. We claim that there exists a maximal anisotropic torus $S$ in $G$ whose Lie algebra is $\mathfrak{s}$. To see this, first observe that since $\mathfrak{s}^{[p]} \subseteq \mathfrak{s},[3$, (4.9)] implies that there does exist a torus $S$ in $G$ having Lie algebra $\mathfrak{s}$ (though we have no assurance that $S$ is $\theta$-stable). Thus, $\mathfrak{s}$ is contained in the Lie algebra of a maximal torus of $G$, and this easily implies that $H:=Z_{G}(\mathfrak{s})^{\circ}$ is a $\theta$-stable reductive subgroup of $G$. Since $S \subseteq H, \mathfrak{s} \subseteq \operatorname{Lie}(H)$. Let $S_{1}$ be a maximal anisotropic torus in $H$. Since $\mathfrak{s}$ is maximal, it follows that $\mathfrak{s}_{1}:=\operatorname{Lie}\left(S_{1}\right) \subseteq \mathfrak{s}$ because $\mathfrak{s}_{1}+\mathfrak{s}$ is a restricted toral subsystem of $\mathfrak{p}$. Now let $H_{1}=Z_{H}\left(S_{1}\right)$. Then $\operatorname{Lie}\left(H_{1}\right)=Z_{\mathfrak{h}}\left(S_{1}\right)$, where $\mathfrak{h}=\operatorname{Lie}(H)$. Thus, $\mathfrak{s} \subseteq \operatorname{Lie}\left(H_{1}\right)$. By [25, (2.6)], if $K_{1}=H_{1}^{\theta}$, then $H_{1}=K_{1}^{\circ} \cdot S_{1}$ (almost direct product). Thus, $\operatorname{Lie}\left(H_{1}\right)=\operatorname{Lie}\left(K_{1}^{\circ}\right) \oplus \mathfrak{s}_{1}$. Since $\theta$ acts trivially on $\operatorname{Lie}\left(K_{1}^{\circ}\right)$ and since $\mathfrak{s}_{1} \subseteq \mathfrak{s}$, it follows that $\mathfrak{s}=\mathfrak{s}_{1}$ so that $\operatorname{Lie}\left(S_{1}\right)=\mathfrak{s}$. Clearly, the anisotropic torus $S_{1}$ is maximal; otherwise, $\mathfrak{s}$ would not be a maximal restricted toral subsystem of $\mathfrak{p}$.

Finally, if $K=G^{\theta}$ (which is connected, since we assume that $G$ is simply connected), any two maximal anisotropic tori in $G$ are $K$-conjugate [25, (2.7)]. Therefore, under the adjoint action of $K$ on $\mathfrak{p}$, it follows that any two maximal restricted toral subsystems of $\mathfrak{p}$ are $K$-conjugate.

\footnotetext{
${ }^{5}$ Suppose $T$ is a finite-dimensional, restricted Lie triple system over $k$ such that $(-)^{[p]}: T \rightarrow T$ is injective. Then $T$ is abelian, and hence a restricted toral Lie triple system. The following proof of this fact is due to Nora Hopkins: Given $0 \neq y \in T$, choose $m$ minimal so that $y^{[p]^{m}}+$ $\alpha_{m-1} y^{[p]^{m-1}}+\cdots+\alpha_{0} y=0, \alpha_{i} \in k$. If $y \neq 0, \alpha_{0} \neq 0$; otherwise, the definitions imply that $\left(y^{[p]^{m-1}}+\alpha_{m-1}^{1 / p} y^{[p]^{m-2}}+\cdots+\alpha_{1}^{1 / p} y\right)^{[p]}=0$ and the injectivity of $(-)^{[p]}$ contradicts the minimality of $m$. Now let $D=D_{a, b} \in \operatorname{Der}(T)$ for $a, b \in T$. Let $0 \neq y \in T$ be an eigenvector for $D$, say $D(y)=\lambda y$ for some $\lambda \in k$. Then by [4.6), $D\left(y^{[p]}\right)=(a, b, y, \ldots, y)=\lambda(y, \ldots, y)=0$ (where $y$ appears $p$-times in the second and third terms). From this it follows easily that $D\left(y^{[p]^{i}}\right)=0$ for any $i>0$. Thus, $-\alpha_{0} \lambda y=D\left(y^{[p]^{m}}+\cdots+\alpha_{1} y^{[p]}\right)=0$. Thus, $\lambda=0$. It follows that the Lie algebra InnDer $(T)$ of inner derivations of $T$ is nilpotent. Hence by Engel's theorem, there exists a nonzero $y \in T$ such that $0=D_{a, b}(y)=[a, b, y]$ for all $a, b \in T$. In particular, for $a \in T$, $(\operatorname{ad} y)^{p}(a)=(-1)^{p}(a, y, y, \ldots, y)=(-1)^{p}\left(D_{a, y}(y), y, \ldots, y\right)=0$. Hence, $(\operatorname{ad} y)^{p}=0$ in $L_{s}(T)$. But using Proposition 4.5, ad $y^{[p]^{r}}=(\operatorname{ad} y)^{p^{r}}$; so ad $y$ satisfies the separable polynomial equation $X^{p^{m}}+\alpha_{m-1} X^{p^{m-1}}+\cdots+\alpha_{0} X=0$. Therefore, ad $y$ is semisimple; so ad $y=0$ and $T$ is abelian.
} 
Having examined some implications of the assumption that a Lie triple system is restricted, we now turn to the determination of the existence of a restricted structure on a given Lie triple system $T$. To begin, an examination of the proof of [17, Lem. 4, p. 189] shows that it generalizes to give the following result.

Lemma 4.10. Let $T$ be a Lie triple system over $k$, and let $(\mathfrak{g}, T$, id $) \in \mathbf{L I E}_{k}(T)$. Suppose there exists a basis $\left\{u_{i}\right\}_{i \in I}$ of $T$ such that, for every $i \in I,\left(\operatorname{ad} u_{i}\right)^{p}=$ ad $v_{i}: \mathfrak{g} \rightarrow \mathfrak{g}$ for some $v_{i} \in T$. The elements $z_{i}:=u_{i}^{p}-v_{i}$ are central in $U(\mathfrak{g})$. If $\left\{w_{j}\right\}_{j \in J}$ is a basis for $[T, T] \subseteq \mathfrak{g}$, upon ordering the sets $I$ and $J$, the elements

$$
z_{i_{1}}^{h_{1}} \cdots z_{i_{r}}^{h_{r}} u_{i_{1}}^{\lambda_{1}} \cdots u_{i_{r}}^{\lambda_{r}} w_{j_{1}}^{m_{1}} \cdots w_{j_{t}}^{m_{t}}
$$

satisfying $i_{1}<\cdots<i_{r}, j_{1}<\cdots<j_{t}, h_{k}, m_{\ell} \geq 0$, and $0 \leq \lambda_{k}<p$, form a basis for $U(\mathfrak{g})$.

Now we can easily obtain the following result, generalizing Theorem 4.1 ,

Theorem 4.11. Let $T$ be a Lie triple system over $k$. Suppose there exists a basis $\left\{u_{i}\right\}_{i \in I}$ of $T$ with the property that for every $i \in I$ there exists a $v_{i} \in T$ such that

$$
\left[a, v_{i}, c\right]=\left(a, u_{i}, \ldots, u_{i}, c\right) \quad\left(p \text { copies of } u_{i}\right), \quad a, c \in T .
$$

Then there is a unique restricted structure $a \mapsto a^{[p]}$ on $T$ such that $u_{i}^{[p]}=v_{i}$ for all $i \in I$.

Proof. By construction of $L_{s}(T)$, the derivations $\left(\operatorname{ad} u_{i}\right)^{p}$ and ad $v_{i}$ of $L_{s}(T)$ agree on $T$ and hence are equal. For $i \in I$, let $z_{i}=u_{i}^{p}-v_{i}$ and let $\left\{w_{j}\right\}_{j \in J}$ be a basis for $[T, T] \subseteq L_{s}(T)$. Then Lemma 4.10 for $\mathfrak{g}=L_{s}(T)$ provides a basis for $U\left(L_{s}(T)\right)$. Let $\mathcal{B}$ be the ideal in $U\left(L_{s}(T)\right)$ generated by the $z_{i}, i \in I$. Then the basis elements $z_{i_{1}}^{h_{1}} \cdots z_{i_{r}}^{h_{r}} u_{i_{1}}^{\lambda_{1}} \cdots u_{i_{r}}^{\lambda_{r}} w_{j_{1}}^{m_{1}} \cdots w_{j_{t}}^{m_{t}}$ for which some $h_{k}>0$ form a basis for $\mathcal{B}$, and the set of cosets in $\bar{U}:=U\left(L_{s}(T)\right) / \mathcal{B}$ of the form $u_{i_{1}}^{\lambda_{1}} \cdots u_{i_{r}}^{\lambda_{r}} w_{j_{1}}^{m_{1}} \cdots w_{j_{t}}^{m_{t}}+\mathcal{B}$, $0 \leq \lambda_{k} \leq(p-1), m_{k} \geq 0$, comprise a basis for $\bar{U}$. In particular, the restriction of the quotient map $U\left(L_{s}(T)\right) \rightarrow \bar{U}$ to $L_{s}(T)$ is an injective Lie algebra morphism. We can therefore regard $L_{s}(T)$ as a Lie subalgebra of $\bar{U}$. In this algebra, $u_{i}^{p}=v_{i} \in L_{s}(T)$ for all $i \in I$. For $x, y \in T$, we have $s_{i}(x, y) \in T$; hence, if $x \in T$, the obvious extension of (4.4b) shows that $x^{p} \in \bar{U}$ actually lies in $L_{s}(T)$. Thus, $x \mapsto x^{[p]}:=x^{p}$, $x \in T$, defines a restricted structure on $T$ which satisfies $u_{i}^{[p]}=v_{i}$. Finally, (4.4b) also shows that the restriction map $(-)^{[p]}$ is completely determined once its values on a basis are given.

A trivial consequence of Theorem 4.11 is:

Corollary 4.12. Let $T$ be a Lie triple system for $k$ and let $(\mathfrak{g}, T$, id $) \in \mathbf{L I E}_{k}(T)$. Suppose there exists a basis $\left\{u_{1}, \ldots, u_{r}\right\}$ of $T$ such that, for $1 \leq i \leq r$, there exists $v_{i} \in T$ with the property that

$$
\left(\operatorname{ad} u_{i}\right)^{p}=\operatorname{ad} v_{i}: \mathfrak{g} \rightarrow \mathfrak{g} .
$$

Then there exists a unique restricted structure $a \mapsto a^{[p]}$ on $T$ such that $u_{i}^{[p]}=v_{i}$, $1 \leq i \leq r$.

The theorem above also implies the following result.

Corollary 4.13. Let $T$ be a Lie triple system over $k$. Suppose that for each $u \in T$, there exists $f(u) \in T$ such that $(\operatorname{ad} u)^{p}=\operatorname{ad} f(u): L_{s}(T) \rightarrow L_{s}(T)$. Then $T$ has a 
restricted structure $x \mapsto x^{[p]}$ which can be chosen to agree with the map $x \mapsto f(x)$ for $x$ belonging to a basis for $T$. Finally, if $T$ has trivial center, then $f$ itself defines a (unique) restricted structure on $T$.

Proof. The only issue to address is the last statement. By [14, Cor. 2.1.8], $Z(T)=0$ if and only if $Z\left(L_{s}(T)\right)=0$. If $a \mapsto a^{[p]}$ and $a \mapsto a^{[p]^{\prime}}$ are two restricted structures on $T$, then by Proposition 4.5, $\left[a^{[p]}, x\right]-\left[a^{[p]^{\prime}}, x\right]=(\operatorname{ad} a)^{p}(x)-(\operatorname{ad} a)^{p}(x)=0$ for all $x \in L_{s}(T)$. Thus, $a^{[p]}-a^{[p]^{\prime}} \in Z\left(L_{s}(T)\right)$, whence $a^{[p]}=a^{[p]^{\prime}}$.

We now define an algebra that will play an important role in the next section of this paper.

Definition 4.14. Let $T$ be a restricted Lie triple system. Let $\mathcal{J}$ be the ideal in $U\left(L_{u}^{[p]}(T)\right)$ generated by the elements $x^{p}-x^{[p]}, x \in T$. Then $U^{[p]}(T)$ will denote the quotient algebra $U\left(L_{u}^{[p]}(T)\right) / \mathcal{J}$. Thus, given any associative algebra $A$ and Lie algebra morphism $\psi: L_{u}^{[p]}(T) \rightarrow A$ satisfying $\psi\left(x^{[p]}\right)=\psi(x)^{p}$, there is a unique algebra morphism $\Psi: U^{[p]}(T) \rightarrow A$ which extends $\psi$.

For the purpose of examining the representation theory of $T$, the preceding definition of $U^{[p]}(T)$ in terms of $L_{u}^{[p]}(T)$ will allow us to draw easy parallels with the nonrestricted case. However, in many cases of interest, $U^{[p]}(T)$ can be characterized more simply as $U^{[p]}(T) \cong U\left(L_{u}(T)\right) / \mathcal{J}^{\prime}$, where $\mathcal{J}^{\prime}$ is the ideal of $U\left(L_{u}(T)\right)$ generated by the elements $x^{p}-x^{[p]}, x \in T$. This occurs, in particular, when $L_{u}(T) \cong L_{s}(T)$, for then by Definition 4.8 and [14, $(2.0 .21)], L_{u}^{[p]}(T) \cong L_{u}(T)$.

Now, returning the viewpoint of Definition 4.14, observe that the map $T \rightarrow$ $U\left(L_{u}^{[p]}(T)\right), x \mapsto x^{P}:=x^{p}-x^{[p]}$, is $p$-linear in the sense that $(\alpha x)^{P}=\alpha^{p} x^{P}$ and $(x+y)^{P}=x^{P}+y^{P}$ for $\alpha \in k, x, y \in T$. Thus, the ideal $\mathcal{J}$ above is also generated by the elements $u_{i}^{p}-u_{i}^{[p]}$ for $\left\{u_{i}\right\}_{i \in I}$ any fixed basis of $T$. In particular, by Lemma 4.10 we have the following result.

Corollary 4.15. Let $T$ be a restricted Lie triple system. Let $\left\{u_{i}\right\}_{i \in I}$ be a basis for $T$ and let $\left\{w_{j}\right\}_{j \in J}$ be a basis for the subspace $[T, T]$ of $L_{u}^{[p]}(T)$. Assume that $I$ and $J$ are ordered. Then the elements $u_{1_{1}}^{m_{1}} \cdots u_{i_{k}}^{m_{k}} w_{j_{1}}^{n_{1}} \cdots w_{j_{\ell}}^{n_{\ell}}$, for $i_{1}<\cdots<i_{k}$, $j_{1}<\cdots<j_{\ell}, 0 \leq m_{s} \leq(p-1)$, and $n_{t} \geq 0$ form a basis for $U^{[p]}(T)$.

Let $T$ be an arbitrary restricted Lie triple system and let $(\mathfrak{g}, T$, id $) \in \mathbf{L I E}_{k}(T)$. A natural question asks if $\mathfrak{g}$ possesses a restricted structure extending that of $T$. Under some hypotheses on $T$ (roughly, that a Killing form analogue for Lie triple systems is nondegenerate), this question has been answered affirmatively in the case $\mathfrak{g}=L_{s}(T)$; for a precise statement, see [14, (3.45)]. In general, $L_{u}(T)$ need not be a restricted Lie algebra with a restricted structure extending that of $T$, although this holds by fiat in a wide class of interesting examples arising from involutions on algebraic group; see Example 4.9(b). On the other hand, let $T$ be toral as in Example 4.9(a), with basis $x_{1}, \ldots, x_{n}$ and restricted structure satisfying $x_{i}^{[p]}=x_{i}$ for all $i$. Then for any $i \neq j, 0 \neq\left[x_{i}, x_{j}\right] \in Z\left(L_{u}(T)\right)$, so that ad $x_{i} \neq 0$. But $\left(\operatorname{ad} x_{i}\right)^{p}$ clearly does equal 0 on $L_{u}(T)$ since $[T, T]=\bigwedge^{2} T$ is central. Thus, $L_{u}(T)$ has no compatible restricted structure extending that of $T$. Here, $L_{u}(T) \varsubsetneqq L_{u}^{[p]}(T)$.

For an arbitrary restricted Lie triple system $T$, the augmentation $\epsilon: U(T) \rightarrow$ $k$ determines an augmentation ${ }^{[p]} \epsilon: U^{[p]}(T) \rightarrow k$ in the obvious manner. Now consider the ideal $\mathcal{J}$ of $U\left(L_{u}^{[p]}(T)\right)$ defined in Definition 4.14. If $\Delta: U\left(L_{u}^{[p]}(T)\right) \rightarrow$ 
$U\left(L_{u}^{[p]}(T)\right) \otimes U\left(L_{u}^{[p]}(T)\right)$ is the comultiplication map on the Hopf algebra $U\left(L_{u}^{[p]}(T)\right)$ and if $x \in T \subset L_{u}^{[p]}(T)$, we have $\Delta\left(x^{p}-x^{[p]}\right)=\Delta(x)^{p}-\Delta\left(x^{[p]}\right)=x^{p} \otimes 1+1 \otimes$ $x^{p}-x^{[p]} \otimes 1-1 \otimes x^{[p]}=\left(x^{p}-x^{[p]}\right) \otimes 1+1 \otimes\left(x^{p}-x^{[p]}\right)$, since $x^{[p]} \in T$ is primitive. As a consequence, $\Delta(\mathcal{J}) \subseteq \mathcal{J} \otimes U(T)+U(T) \otimes \mathcal{J}$. In addition, if $\epsilon$ and $\gamma$ denote the counit and antipode on $U\left(L_{u}^{[p]}(T)\right)$, then $\epsilon(\mathcal{J})=0$ and $\gamma(\mathcal{J}) \subseteq \mathcal{J}$. Thus, $\mathcal{J}$ is a Hopf ideal, and $U^{[p]}(T)$ inherits a unique Hopf algebra structure from that of $U\left(L_{u}^{[p]}(T)\right)$.

The involution $\theta$ on $L_{u}^{[p]}(T)$ extends to an involution $\theta$ on $U\left(L_{u}^{[p]}(T)\right)$. For $x \in T, \theta\left(x^{p}-x^{[p]}\right)=-\left(x^{p}-x^{[p]}\right) \in \mathcal{J}$; so $\theta(\mathcal{J})=\mathcal{J}$. Thus, $\theta$ induces an involution on $U^{[p]}(T)$, which is easily checked to be a Hopf algebra involution. Following Lemma 2.6 we can consider the (cocommutative) Hopf algebra $K^{[p]}(T):=$ $K\left(U^{[p]}(T), \theta\right)$. We also write ${ }^{[p]} \epsilon^{ \pm}: K^{[p]}(T) \rightarrow k^{ \pm}$for the maps $\epsilon^{ \pm}: K\left(U^{[p]}(T), \theta\right)$ $\rightarrow k^{ \pm}$. Of course, we have the isomorphism $\left(U^{[p]}(T), \theta\right)-$ Mod $\cong K^{[p]}(T)-$ Mod, where $\left(U^{[p]}(T), \theta\right)$-Mod denotes the category of objects in $U^{[p]}(T)$-Mod that have a compatible action of $\theta$.

We complete this section by considering the full subcategory $L_{u}^{[p]}(T)-\operatorname{Mod}_{[p]}$ of $L_{u}^{[p]}(T)$-Mod consisting of all $L_{u}^{[p]}(T)$-modules $V$ with the property that, for any $x \in T$, the corresponding linear operators $x_{V}$ and $x_{V}^{[p]}$ on $V$ satisfy the identity

$$
x_{V}^{[p]}=\left(x_{V}\right)^{p} .
$$

There is an equivalence $L_{u}^{[p]}(T)-\operatorname{Mod}_{[p]} \cong U^{[p]}(T)-\mathbf{M o d}$ of categories. Now let $\left(L_{u}^{[p]}(T), \theta\right)-\mathbf{M o d}_{[p]}$ denote the category consisting of objects $V \in L_{u}^{[p]}(T)-\mathbf{M o d}_{[p]}$ that have a compatible action of $\theta$. Clearly,

$$
\left(L_{u}^{[p]}(T), \theta\right)-\operatorname{Mod}_{[p]} \cong\left(U^{[p]}(T), \theta\right)-\mathbf{M o d} \cong K^{[p]}(T)-\mathbf{M o d}
$$

These categories will be instrumental in the next section.

\section{Modules for Restricted Lie triple Systems}

In this section, we maintain the notation of the previous section. Given a restricted Lie triple system $T$, we now develop the notion of a restricted $T$-module.

Definition 5.1. Let $M$ be a module for a restricted Lie triple system $T$. Then $M$ is restricted provided that

$$
\left[a, b^{[p]}, c\right]=(a, b, \ldots, b, c)(p \text { copies of } b)
$$

for all $b \in T$ and $a, c \in E_{M} 6$

By linearity in $a, c$, the identity in Definition 5.1 needs to be checked only when $a, c \in T \cup M$. Of course, $\left[a, b^{[p]}, c\right]=0$ if both $a, c \in M$. If $M$ is restricted, then $\left[a^{[p]}, b, c\right]$, for $a \in T, b, c \in E_{M}$, is determined by the identity $\left[a^{[p]}, b, c\right]=-\left[b, a^{[p]}, c\right]$. If $c \in T, a, b \in E_{M}$, then, working in $L_{s}\left(E_{M}\right)$, the argument given for (4.6) shows that $\left[a, b, c^{[p]}\right]=(a, b, c, \ldots, c)(p$ copies of $c)$.

\footnotetext{
${ }^{6}$ Suppose that $\mathfrak{g}$ is a restricted Lie algebra and $V$ is a restricted $\mathfrak{g}$-module. On the split extension $\widetilde{\mathfrak{g}}=\mathfrak{g} \oplus V$, define $(-)^{[p]}: \widetilde{\mathfrak{g}} \rightarrow \widetilde{\mathfrak{g}}$ by $(a+v)^{[p]}=a^{[p]}+\sum_{i} s_{i}(a, v)=a^{[p]}+(a, v, a, \ldots, a)$ for $a \in \mathfrak{g}$ and $v \in V$. In general, $(-)^{[p]}$ does not define a restricted structure on $\tilde{\mathfrak{g}}$. Similarly, if $M$ is a restricted module for a restricted Lie triple system, then $E_{M}$ does not have a corresponding restricted Lie triple system structure.
} 
By definition, a morphism $\psi: M \rightarrow M^{\prime}$ of restricted $T$-modules is just a linear map that defines a morphism in $T$-Mod. Let $T-\mathbf{M o d}_{[p]}$ denote the category of restricted $T$-modules, and let $F: T-\mathbf{M o d}_{[p]} \rightarrow T$-Mod be the evident forgetful functor. The functor $F$ is a full and faithful embedding, and it will be convenient to regard $T-\operatorname{Mod}_{[p]}$ as a (strict) full subcategory of $T-$ Mod.

We will now indicate how the ideas of $\$ 3$ can be adapted to the study of the category of restricted $T$-modules.

Proposition 5.2. Let $T$ be a restricted Lie triple system, and $N \in K^{[p]}(T)-$ Mod. Then the -1-eigenspace $N^{-}=\{n \in N \mid \theta(n)=-n\}$ is naturally a restricted $T$ module.

Proof. Recalling the end of $₫ 4$ we may regard $N$ as a module in $\left(L_{u}^{[p]}(T), \theta\right)-\operatorname{Mod}_{[p]}$; so $N$ is trivially an $\left(L_{u}(T), \theta\right)$-module. Thus, $N^{-} \in T-$ Mod. For $b \in T, b^{[p]}=b^{p}$ in $K^{[p]}(T)$. Using this equality, the required identity in Definition 5.1 follows immediately for $M=N^{-}$.

By Proposition 5.2, there is an exact functor ${ }^{[p]} j^{*}: K^{[p]}(T)-\operatorname{Mod} \rightarrow T-\mathbf{M o d}_{[p]}$ obtained by setting ${ }^{[p]} j^{*} N=N^{-}$. Regarding $T-\operatorname{Mod}_{[p]}$ as a full subcategory of $T$-Mod, it will sometimes be convenient to think of $[p] j^{*}$ as a functor $K^{[p]}(T)$-Mod $\rightarrow T-\mathbf{M o d}$ which has image in $T-\operatorname{Mod}_{[p]}$. Clearly, $\mathcal{S}=\operatorname{Ker}\left({ }^{[p]} j^{*}\right)$ identifies with all $N \in K^{[p]}(T)$-Mod n such that $\theta$, and hence $T$, act trivially. Equivalently, $N \in \mathcal{S}$ if and only if $K^{[p]}(T)$ acts through its augmentation ${ }^{[p]} \epsilon^{+}: K^{[p]}(T) \rightarrow k$. As in $\S 3, \mathcal{S} \cong \mathbf{V e c}_{k}$ is a Serre subcategory of $K^{[p]}(T)$-Mod. Let ${ }^{[p]} i_{*}: \mathbf{V e c}_{k} \rightarrow$ $K^{[p]}(T)$-Mod be the natural full embedding that associates to any vector space $V$ its natural structure as a trivial $K^{[p]}(T)$-module. As with $i_{*}: \mathbf{V e c}_{k} \rightarrow K(T)$-Mod, there is an adjoint triple $\left({ }^{[p]} i^{*},{ }^{[p]} i_{*},{ }^{[p]} i^{!}\right)$in which, given $M \in K^{[p]}(T),{ }^{[p]} i^{*} M$ (resp., $\left.{ }_{[p]} i^{!} M\right)$ is the largest trivial quotient module (resp., submodule) of $M$.

By construction, the algebra $U^{[p]}(T)$ is a quotient algebra of $U(T)$; thus, there is a surjective algebra morphism $K(T) \stackrel{\pi}{\rightarrow} K^{[p]}(T)$, and we let $I_{*}: K^{[p]}(T)-\operatorname{Mod} \rightarrow$ $K(T)$-Mod be the corresponding inflation functor, in which a $K^{[p]}(T)$-module is regarded as a $K(T)$-module through the morphism $\pi$. Further, we have the diagram

$$
K^{[p]}(T)-\operatorname{Mod} \underset{I^{!}}{\stackrel{I_{*}^{*}}{\longrightarrow}} K(T)-\operatorname{Mod} \underset{j_{*}}{\stackrel{j^{*}}{\longrightarrow}} T-\operatorname{Mod} \stackrel{5}{\longleftarrow} T-\operatorname{Mod}_{[p]} .
$$

Here $\left(I^{*}, I_{*}, I^{!}\right)$is an adjoint triple: for $M \in K(T)-\operatorname{Mod}, I^{*} M\left(\operatorname{resp} ., I^{!} M\right)$ is the largest quotient module (resp., submodule) of $M$ that is a $K^{[p]}(T)$-module. Since $j^{*} I_{*}$ has image in $T-\operatorname{Mod}_{[p]}$ and, in fact, identifies with ${ }^{[p]} j^{*}$, it is immediate that ${ }^{[p]} j_{!}=I^{*} j_{!} F: T-\operatorname{Mod}_{[p]} \rightarrow K^{[p]}(T)-\operatorname{Mod}$ defines a left adjoint to ${ }^{[p]} j^{*}$. Similarly, ${ }^{[p]} j_{*}=I^{!} j_{*} F$ is right adjoint to ${ }^{[p]} j^{*}$.

With this discussion, we can now state the following fundamental result.

Theorem 5.4. Let $T$ be a restricted Lie triple system, and let ${ }^{[p]} j^{*}: K^{[p]}(T)-\mathbf{M o d}$ $\rightarrow T-\operatorname{Mod}_{[p]}$, etc., be as above.

(a) $T-\operatorname{Mod}_{[p]}$ is isomorphic to the quotient category $K^{[p]}(T)-\operatorname{Mod} / \mathcal{S}$, for $\mathcal{S}=$ $\operatorname{Ker}\left({ }^{[p]} j^{*}\right) \cong \operatorname{Vec}_{k}$. 
(b) There is a diagram

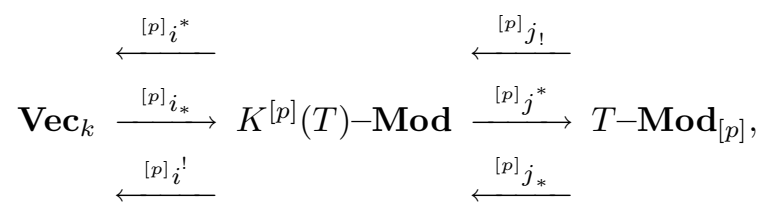

with adjoint triples $\left({ }^{[p]} i^{*},{ }^{[p]} i_{*},{ }^{[p]} i^{!}\right)$and $\left({ }^{[p]} j_{!},{ }^{[p]} j^{*},{ }^{[p]} j_{*}\right)$ as defined above. We have $[p] i^{*}[p] j_{!}=0=[p] i^{!}[p] j_{*}$. Also, ${ }^{[p]} i^{*}[p] i_{*} \cong\left[{ }^{[p]} i^{!}[p] i_{*} \cong\right.$ id and ${ }^{[p]} j^{*}[p] j_{!} \cong[p] j^{*}[p] j_{*} \cong$ id .

(c) Consider the idempotent $e=\frac{1-\theta}{2} \in K^{[p]}(T)$. There is another diagram

$$
K^{[p]}(T) / K^{[p]}(T) e K^{[p]}(T)-\mathbf{M o d} \stackrel{\stackrel{[p] i^{*}}{\stackrel{[p] i_{*}}{\longrightarrow}}}{\stackrel{[p] i}{\longleftarrow}} K^{[p]}(T)-\mathbf{M o d} \stackrel{\stackrel{[p] j_{1}}{\stackrel{[p] j^{*}}{\longrightarrow}} \text { e }}{\stackrel{[p] j_{*}}{\longleftarrow}} \text { K } K^{[p]}(T) e-\mathbf{M o d}
$$

in which $\left([p] i^{*},{ }^{[p]} i_{*},{ }^{[p]} i^{!}\right)$and $\left({ }^{[p]} j_{!},{ }^{[p]} j^{*},{ }^{[p]} j_{*}\right)$ are adjoint triples satisfying ${ }^{[p]} i^{*}[p] j_{!}$ $=0={ }^{[p]} i^{!}[p] j_{*},{ }^{[p]} i^{*}[p] i_{*} \cong[p] i^{!}[p] i_{*} \cong \mathrm{id}$, and ${ }^{[p]} j^{*}[p] j_{!} \cong[p] j^{*}[p] j_{*} \cong \mathrm{id}$. Then $e K^{[p]}(T) e-\mathbf{M o d} \cong T-\mathbf{M o d}_{[p]}$ and $K^{[p]}(T) / K^{[p]}(T) e K^{[p]}(T)-\mathbf{M o d} \cong \operatorname{Vec}_{k}$. With these identifications, (5.4.2) is identical to (5.4.1).

(d) The category $T-\mathbf{M o d}_{[p]}$ has enough injectives and enough projectives. Every object in $T-\mathbf{M o d}_{[p]}$ has an injective envelope.

(e) Let $U^{[p]}(T)^{\theta}=\left\{a \in U^{[p]}(T) \mid \theta(a)=a\right\}$. Then $U^{[p]}(T)^{\theta}-\mathbf{M o d} \cong T-\operatorname{Mod}_{[p]}$.

Proof. The argument follows along lines similar to those given in $\S 3$, but differs in some respects. First, we show that ${ }^{[p]} j^{*}[p] j_{*} \cong \mathrm{id}$. For $M \in T-\mathbf{M o d}_{[p]}$, recall that $j_{*}^{\prime} M$ is the submodule of $L_{s}\left(E_{M}\right)$ generated (as a $L_{u}(T)$-module) by $M$. By the definition of $L_{s}\left(E_{M}\right)$, it follows that $j_{*}^{\prime} M \in K^{[p]}(T)$-Mod already, since $M$ is a restricted module. Now $j_{*}^{\prime} M \subseteq j_{*} M$, while ${ }^{[p]} j_{*} M=I^{!} j_{*} M$ is the largest $K(T)$-submodule of $j_{*} M$ that is a $K^{[p]}(T)$-module. Thus, $j_{*}^{\prime} M \subseteq\left[{ }^{[p]} j_{*} M\right.$. Also, ${ }^{[p]} j_{*} M / j_{*}^{\prime} M$ is a trivial $K(T)$-module, hence a trivial $K^{[p]}(T)$-module. Therefore, $[p] j^{*}[p] j_{*} M=M$, as required.

Hence, ${ }^{[p]} j_{*}$ is a section for ${ }^{[p]} j^{*}$, and by $[6,(15.19)],{ }^{[p]} j^{*}$ is a quotient morphism with kernel the Serre subcategory $\mathcal{S}$ consisting of all trivial $K^{[p]}(T)$-modules. Everything in (a) and (b) is clear, except the assertion that ${ }^{[p]} j^{*}[p] j_{!} \cong \mathrm{id}$, which we will show next in the course of proving (c).

Now let $e \in K^{[p]}(T)$ be the idempotent given in (c). Then from general principles we have a diagram as stated in (c) in which the functors there satisfy ${ }^{[p]} i^{*}[p] j_{!}=0=$ ${ }_{[p]} i^{!}[p] j_{*},[p] i^{*}[p] i_{*} \cong[p] i_{i}{ }^{[}[p] i_{*} \cong \mathrm{id}$, and ${ }^{[p]} j^{*}[p] j_{!} \cong[p] j^{*}[p] j_{*} \cong \mathrm{id}$. In this diagram, a $K^{[p]}(T)$-module $M$ is annihilated by ${ }^{[p]} j^{*}$ if and only if $e M=0$. But $e M=0$ if and only if $\theta$ acts trivially on $M$, i.e., $M \in \mathcal{S}$. This proves (c) and completes the proof of (b) since the left adjoint ${ }^{[p]} j_{1}$ in (5.4.1) must identify with the left adjoint in $(5.4 .2)$ which does satisfy $[p] j^{*}[p] j_{!} \cong \mathrm{id}$.

Next, (d) follows from (c), since the category $e K^{[p]}(T) e-$ Mod has enough projectives and injectives, and also has injective envelopes. 
Finally, analogous to the nonrestricted case, $e K^{[p]}(T) e \cong U^{[p]}(T)^{\theta}$; so (e) is an immediate consequence of (c).

Example 5.5. Let $T$ be a toral Lie triple system (which is, by definition, finitedimensional). By Example $4.9 T$ has a basis $u_{1}, \ldots, u_{m}$ satisfying $u_{i}^{[p]}=u_{i}$ for all $i$. Thus, in $L_{u}(T), \operatorname{ad} u_{i}^{[p]}-\operatorname{ad}\left(u_{i}\right)^{p}=\operatorname{ad} u_{i}$; so $L_{u}^{[p]}(T)=T$, regarded as an abelian Lie algebra that has an induced restricted structure. By [17, Thm. 14, p. 193], any finite-dimensional restricted $L_{u}^{[p]}(T)$-module is completely reducible, isomorphic to a direct sum of one-dimensional submodules defined by linear functionals $\phi: T \rightarrow k$ satisfying $\phi\left(u_{i}\right)^{p}=\phi\left(u_{i}\right) \in \mathbb{F}_{p}$. Thus, any finite-dimensional restricted module $V$ for the Lie triple system $T$ is completely reducible into one-dimensional submodules. Equivalently, $V$ has a basis $v_{1}, \ldots, v_{n}$ such that $\left[v_{j}, s, t\right]=\phi_{j}(s) \phi_{j}(t) v_{j}$ for all $j$ and $s, t \in T$. Here $\phi_{j}: T \rightarrow k$ satisfies $\phi_{j}\left(u_{i}\right)^{p}=\phi_{j}\left(u_{i}\right)$ for all $i$.

We conclude this section with some remarks on the irreducible modules in $T-\operatorname{Mod}_{[p]}$.

Proposition 5.6. Let $T$ be a finite-dimensional Lie triple system over $k$. Then:

(a) The irreducible $T$-modules are finite-dimensional. If $T$ is restricted, the irreducible restricted $T$-modules are finite-dimensional.

(b) Assume that $T$ is restricted. Then the functor $[p] j^{*}$ maps the distinct, nontrivial irreducible $K^{[p]}(T)$-modules bijectively to the distinct restricted irreducible T-modules.

Proof. Because $T$ is finite-dimensional, $L_{u}(T)$ is also finite dimensional. A standard argument given in [10, p. 1057] shows that any irreducible $L_{u}(T)$-module is finitedimensional. Given an irreducible $\left(L_{u}(T), \theta\right)$-module $V$ (i.e., an irreducible $K(T)$ module), another standard argument shows that either $V$ is an irreducible $L_{u}(T)$ module, or $V=M \oplus \theta(M)$ for an irreducible $L_{u}(T)$-submodule $M$. In either case, $V$ is finite-dimensional. This proves (a), while (b) follows in the same fashion as Corollary 3.7.

Suppose $G$ is a simple, simply connected group over $k$ and $T=\mathfrak{p}$ is obtained from an involution $\theta$. For simplicity, assume that $L_{u}(\mathfrak{p})=L_{s}(\mathfrak{p})=\mathfrak{g}$. In this case, there is considerable information on the irreducible $\mathfrak{g}$-modules; e.g., see [10].

\section{Cohomology of Lie triple Systems}

In this section, unless further specified, the algebraically closed field $k$ has any characteristic char $(k) \neq 2$. We give a brief account of some formal aspects of the cohomology in the category $T$-Mod for a Lie triple system $T$. Having carried out a thorough examination of the structure of $T$-Mod and the accompanying category $T-\operatorname{Mod}_{[p]}$, in the case when $T$ is restricted, which highlights the analogies between these two categories, we will not treat restricted cohomology in great detail here, with the exception of some comments leading up to the definition of "Hochschild maps" in 6.5. For the most part, it suffices to say that many of the results for nonrestricted cohomology below apply to the category of $T-\mathbf{M o d}_{[p]}$. In particular, the results of Proposition 6.3 and Definition 6.4 all carry through, replacing $K(T)-\operatorname{Mod}$ by $K^{[p]}(T)-\mathbf{M o d}$ and working in the category $T-\operatorname{Mod}_{[p]}$. 
6.1. Some preliminaries. Let $T$ be a Lie triple system over $k$. For any $M, N \in$ $T$-Mod, the groups $\operatorname{Ext}_{T}^{\bullet}(M, N)$ can be computed using either a projective resolution of $M$ or an injective resolution of $N$. In particular, letting $k \in T-\mathbf{M o d}$ denote the trivial $T$-module, we put $H^{\bullet}(T, M)=\operatorname{Ext}_{T}^{\bullet}(k, M)$. Thus, for any nonnegative integer $n, H^{n}(T, M)$ is the $n$ th-right derived functor $R^{n}(-)^{T}$ evaluated at $M$ of the fixed point functor $(-)^{T}: T-\mathbf{M o d} \rightarrow \mathbf{V e c}_{k}$. Specifically, $M^{T}=\{m \in M \mid[a, m, b]=0=[a, b, m], \forall a, b \in T\}$. The following result provides several simple characterizations of $M^{T}$.

Proposition 6.1.1. Let $M \in T$-Mod. Then $M^{T} \cong\left(j_{*}^{\prime} M\right)^{L_{u}(T)} \cong\left(j_{*} M\right)^{L_{u}(T)}$. Also

$$
M^{T} \cong \operatorname{Hom}_{K(T)}\left(k^{-}, j_{*}^{\prime} M\right) \cong \operatorname{Hom}_{K(T)}\left(k^{-}, j_{*} M\right)
$$

Proof. First, observe that $\left(j_{*} M\right)^{L_{u}(T)}$ is $\theta$-stable. By Theorem $3.9(\mathrm{a}),\left.\theta\right|_{\left(j_{*} M\right)^{L_{u}(T)}}$ $=-\mathrm{id}$. Hence

$$
\begin{aligned}
\left(j_{*} M\right)^{L_{u}(T)} & \cong \operatorname{Hom}_{L_{u}(T)}\left(k, j_{*} M\right) \\
& \cong \operatorname{Hom}_{K(T)}\left(k^{-}, j_{*} M\right) \\
& \cong \operatorname{Hom}_{T}\left(j^{*} k^{-}, M\right) \\
& \cong \operatorname{Hom}_{T}(k, M)=M^{T}
\end{aligned}
$$

Similarly, $\left(j_{*}^{\prime} M\right)^{L_{u}(T)} \cong \operatorname{Hom}_{K(T)}\left(k^{-}, j_{*}^{\prime} M\right)$. By Proposition 3.2 and Theorem 3.9(b), there is a short exact sequence

$$
0 \rightarrow j_{*}^{\prime} M \rightarrow j_{*} M \rightarrow i_{*} V \rightarrow 0
$$

for some $V \in \operatorname{Vec}_{k}$. Since $\operatorname{Hom}_{K(T)}\left(k^{-}, i_{*} V\right)=0$,

$$
\operatorname{Hom}_{K(T)}\left(k^{-}, j_{*}^{\prime} M\right) \cong \operatorname{Hom}_{K(T)}\left(k^{-}, j_{*} M\right) .
$$

6.2. Harris cohomology. Consider a pair $(\mathfrak{g}, \theta)$ consisting of a Lie algebra $\mathfrak{g}$ and involution $\theta$. Given $V \in \mathfrak{g}-\operatorname{Mod}$, the cohomology groups $H^{\bullet}(\mathfrak{g}, V) \cong \operatorname{Ext}_{U(\mathfrak{g})}^{\bullet}(k, V)$ can be computed in terms of the standard (Chevalley-Eilenberg) projective resolution $E_{\bullet}=U(\mathfrak{g}) \otimes \Lambda^{\bullet} \mathfrak{g} \rightarrow k$ of the trivial module $k$. Here $\Lambda^{\bullet} \mathfrak{g}$ is the exterior algebra based on $\mathfrak{g}$. For $n \geq 1$, the differential $d_{n}: E_{n} \rightarrow E_{n-1}$ is defined by

$$
\begin{aligned}
d_{n}(a & \left.\otimes x_{1} \wedge \cdots \wedge x_{n}\right)=\sum_{i=1}^{n}(-1)^{i+1} a x_{i} \otimes x_{1} \wedge \cdots \wedge \widehat{x_{i}} \wedge \cdots \wedge x_{n} \\
& +\sum_{i<j}(-1)^{i+j} a \otimes\left[x_{i}, x_{j}\right] \wedge x_{1} \wedge \cdots \wedge \widehat{x_{i}} \wedge \cdots \wedge \widehat{x_{j}} \wedge \cdots \wedge x_{n}
\end{aligned}
$$

while $d_{0}=\epsilon: U(T) \rightarrow k$ is the augmentation map on $E_{0}=U(\mathfrak{g})=U(\mathfrak{g}) \otimes \bigwedge^{0} \mathfrak{g}$. Now

$$
C^{n}(V)=\operatorname{Hom}_{U(\mathfrak{g})}\left(E_{n}, V\right) \cong \operatorname{Hom}\left(\bigwedge^{n} \mathfrak{g}, V\right)
$$


is the space of alternating multilinear functions $\mathfrak{g}^{\times n} \rightarrow V$. If $\left\{C^{\bullet}(V), \delta^{\bullet}\right\}$ is the complex defined by taking

$$
\begin{aligned}
\delta^{n}(f)\left(x_{1}, \ldots, x_{n+1}\right)= & \sum_{i=1}^{n+1}(-1)^{i+1} x_{i} f\left(x_{1}, \ldots, \widehat{x_{i}}, \ldots, x_{n+1}\right) \\
& +\sum_{i<j}(-1)^{i+j} f\left(\left[x_{i}, x_{j}\right], x_{1}, \ldots, \widehat{x_{i}}, \ldots, \widehat{x_{j}}, \ldots, x_{n+1}\right),
\end{aligned}
$$

then $H^{\bullet}(\mathfrak{g}, V)$ is the cohomology $H^{\bullet}\left(C^{\bullet}(V), \delta^{\bullet}\right)$ of this complex.

Now suppose that $V \in(\mathfrak{g}, \theta)-\mathbf{M o d}$, so that $V$ has an action of $\theta$ compatible with that of $\mathfrak{g}$. The action of $\theta$ extends naturally to a graded action on $C^{\bullet}(V)$ which commutes with the differentials $\delta^{\bullet}$. Thus, as a complex, $C^{\bullet}(V)$ has the decomposition $C^{\bullet}(V)=C \bullet(V)_{+} \oplus C^{\bullet}(V)_{-}$into +1- and -1-eigenspaces for $\theta$. Specifically, $C^{n}(V)_{+}$consists of those multilinear alternating functions $f: \mathfrak{g}^{\times n} \rightarrow V$ satisfying

$$
f\left(\theta\left(x_{1}\right), \ldots, \theta\left(x_{n}\right)\right)=\theta\left(f\left(x_{1}, \ldots, x_{n}\right)\right),
$$

while $C^{n}(V)$ - consists of those $f$ that satisfy

$$
f\left(\theta\left(x_{1}\right), \ldots, \theta\left(x_{n}\right)\right)=-\theta\left(f\left(x_{1}, \ldots, x_{n}\right)\right) .
$$

This leads to a corresponding action of $\theta$ on $H^{\bullet}(\mathfrak{g}, V)$ and a decomposition

$$
H^{\bullet}(\mathfrak{g}, V)=H_{+}^{\bullet}(\mathfrak{g}, V) \oplus H_{-}^{\bullet}(\mathfrak{g}, V)
$$

into +1 - and -1 -eigenspaces for $\theta$.

Example 6.2.1. Let $G$ be a simple, simply connected algebraic group over $k$ of rank $\ell$. Assume that either $k$ has characteristic 0 , or has characteristic $p \geq 3 h-3$, where $h$ is the Coxeter number of $G$. Then

$$
H^{\bullet}(\mathfrak{g}, k) \cong\left(\bigwedge^{\bullet} \mathfrak{g}^{*}\right)^{G}=\left(\bigwedge^{\bullet} \mathfrak{g}^{*}\right)^{\mathfrak{g}} \cong \bigwedge^{\bullet} P,
$$

where $P$ is a vector space of dimension $\ell$, which is graded in degrees $2 m_{1}+$ $1, \ldots, 2 m_{\ell}+1$, where $m_{1}, \ldots, m_{\ell}$ are the exponents of the Weyl group $W$ of $G$. (See [7] and [1].)

Now assume that $\theta$ is an involution on $G$. The action of $\theta$ on $\mathfrak{g}$ induces an action of $\theta$ on $P$. In case $\theta$ is inner, the above isomorphisms imply that $H^{\bullet}(\mathfrak{g}, k)=$ $H_{+}^{\bullet}\left(\mathfrak{g}, k^{+}\right) \cong H_{-}^{\bullet}\left(\mathfrak{g}, k^{-}\right)$. In general, when $\theta$ is an outer automorphism, it need not act trivially on $P$. So if $P=P_{+} \oplus P_{-}$is the decomposition of $P$ into +1 - and -1-eigenspaces, then

$$
H_{+}^{\bullet}\left(\mathfrak{g}, k^{+}\right) \cong \bigwedge^{\bullet} P_{+} \otimes \bigwedge^{\text {even }} P_{-}, \quad H_{-}^{\bullet}\left(\mathfrak{g}, k^{+}\right) \cong \bigwedge^{\bullet} P_{+} \otimes \bigwedge^{\text {odd }} P_{-} .
$$

Also, $H_{\mp}^{\bullet}\left(\mathfrak{g}, k^{-}\right) \cong H_{ \pm}^{\bullet}\left(\mathfrak{g}, k^{+}\right)$.

Return now to an arbitrary pair $(\mathfrak{g}, \theta)$. As at the end of 2, form the algebra $K=K(\mathfrak{g}, \theta)=U(\mathfrak{g}) \oplus U(\mathfrak{g}) \theta$; then $K-\mathbf{M o d} \cong(\mathfrak{g}, \theta)$-Mod. We have $K \otimes_{U(\mathfrak{g})} k \cong$ $k^{+} \oplus k^{-}$. Thus,

$$
Y_{\bullet}=K \otimes_{U(\mathfrak{g})} E_{\bullet} \stackrel{\epsilon^{+} \oplus \epsilon^{-}}{\rightarrow} k^{+} \oplus k^{-}
$$

provides a $K$-projective resolution of $k^{+} \oplus k^{-}$. Of course, $Y_{\bullet} \cong K \otimes \Lambda^{\bullet}(\mathfrak{g})$. 
Let $\bigwedge_{-}^{n}=\left\{\lambda_{n} \in \bigwedge^{n} \mathfrak{g} \mid \theta\left(\lambda_{n}\right)=-\lambda_{n}\right\}$ and $\bigwedge_{+}^{n}=\left\{\lambda_{n} \in \bigwedge^{n} \mathfrak{g} \mid \theta\left(\lambda_{n}\right)=\lambda_{n}\right\}$. If

$$
Y_{n}^{+}=\left\{a \otimes \lambda_{n}+a \theta \otimes \theta\left(\lambda_{n}\right) \mid a \in U(\mathfrak{g}), \lambda_{n} \in \bigwedge^{n} \mathfrak{g}\right\}
$$

and

$$
Y_{n}^{-}=\left\{a \otimes \lambda_{n}-a \theta \otimes \theta\left(\lambda_{n}\right) \mid a \in U(\mathfrak{g}), \lambda_{n} \in \bigwedge^{n} \mathfrak{g}\right\},
$$

then

$$
Y_{n}^{+}=\left(K f \otimes \bigwedge_{+}^{n}\right) \oplus\left(K e \otimes \bigwedge_{-}^{n}\right), \quad Y_{n}^{-}=\left(K f \otimes \bigwedge_{-}^{n}\right) \oplus\left(K e \otimes \bigwedge_{+}^{n}\right),
$$

where $e$ and $f$ are defined as in (3.11). The differential $d_{\bullet}$ on $Y_{\bullet}$ restricts to give complexes $\left(Y_{\bullet}^{+}, d_{\bullet}^{+}\right)$and $\left(Y_{\bullet}^{-}, d_{\bullet}^{-}\right)$. Then $Y_{\bullet}^{+} \stackrel{\epsilon^{+}}{\rightarrow} k^{+}$and $Y_{\bullet}^{-} \stackrel{\epsilon^{-}}{\rightarrow} k^{-}$are $K-$ projective resolutions. For any $K$-module $V$, we have subcomplexes $\left(X_{ \pm}^{\bullet}(V), \delta_{ \pm}^{\bullet}\right)$ of $\left(\operatorname{Hom}_{K}\left(Y_{\bullet}, V\right), \delta^{\bullet}\right)$, where

$$
X_{+}^{\bullet}(V)=\operatorname{Hom}_{K}\left(Y_{\bullet}^{+}, V\right), \quad X_{-}^{\bullet}(V)=\operatorname{Hom}_{K}\left(Y_{\bullet}^{-}, V\right) .
$$

The cohomology of $X_{+}^{\bullet}(V)$ (respectively, $X_{-}^{\bullet}(V)$ ) computes $\operatorname{Ext}_{K}{ }^{\bullet}\left(k^{+}, V\right)$ (respectively, $\left.\operatorname{Ext}_{K}^{\bullet}\left(k^{-}, V\right)\right)$.

Proposition 6.2.3. Let $(\mathfrak{g}, \theta)$ be a Lie algebra with involution. We have

$$
H_{+}^{\bullet}(\mathfrak{g}, V) \cong \operatorname{Ext}_{K}^{\bullet}\left(k^{+}, V\right), \quad H_{-}^{\bullet}(\mathfrak{g}, V) \cong \operatorname{Ext}_{K}^{\bullet}\left(k^{-}, V\right) .
$$

Now let $T$ be a Lie triple system. We can apply the above set-up to the situation $(\mathfrak{g}, \theta)=\left(L_{u}(T), \theta\right)$. Given $M \in T-\mathbf{M o d}$, recall the standard extension $j_{*}^{\prime} M$ defined in (3.1). We have the following notion of the Harris cohomology of $T$. In particular, the groups $H_{B H,+}^{\bullet}(T, M)$ are introduced in 12 .

Definition 6.2.4. For $M \in T$-Mod, define

$$
H_{B H,+}^{\bullet}(T, M)=\operatorname{Ext}_{K(T)}^{\bullet}\left(k^{+}, j_{*}^{\prime} M\right), \quad H_{B H,-}^{\bullet}(T, M)=\operatorname{Ext}_{K(T)}^{\bullet}\left(k^{-}, j_{*}^{\prime} M\right) .
$$

More generally, given a pair $(\mathfrak{g}, \theta)$ as above and $V \in(\mathfrak{g}, \theta)-\mathbf{M o d}$, define

$$
H_{B H, \pm}^{\bullet}(\mathfrak{g}, V)=\operatorname{Ext}_{K}^{\bullet}\left(k^{ \pm}, V\right) .
$$

Given $V \in \mathfrak{g}$-Mod, let $\operatorname{Der}(\mathfrak{g}, V)$ be the space of all linear maps $D: \mathfrak{g} \rightarrow V$ satisfying $D([x, y])=x D(y)-y D(x), x, y \in \mathfrak{g}$. If, in addition, $V \in(\mathfrak{g}, \theta)-\mathbf{M o d}$, then $\theta$ acts on $\operatorname{Der}(\mathfrak{g}, V)$ by $(\theta D)(x)=\theta(D(\theta(x)), x \in \mathfrak{g}$. Let InnDer $(\mathfrak{g}, V)$ be the space of those derivations $D \in \operatorname{Der}(\mathfrak{g}, V)$ for which there exists $v \in V$ such that $D(x)=x \cdot v, x \in \mathfrak{g}$.

Most of the main results in 12] can be summarized in the two results below.

Theorem 6.2.5 ([12]). Let $(\mathfrak{g}, \theta)$ be a Lie algebra with involution and $V \in$ $(\mathfrak{g}, \theta)-$ Mod. Then:

(a) $H_{B H,+}^{0}(\mathfrak{g}, V)=e V \cap V^{\mathfrak{g}}$, where $e=\frac{1-\theta}{2}$ as in (3.11). (Observe $\mathrm{eV}=i_{*} i_{!} V$.)

(b) $H_{B H,+}^{1}(\mathfrak{g}, V)=\operatorname{Der}(\mathfrak{g}, V)^{\theta} / I$, where $I=\{D \in \operatorname{InnDer}(\mathfrak{g}, V) \mid \exists v \in \mathrm{eV}$ such that $D(x)=x \cdot v\}$.

(c) $H_{B H,+}^{2}(\mathfrak{g}, V)$ is in one-to-one correspondence with equivalence classes of central extensions

$$
0 \rightarrow V \stackrel{\iota}{\rightarrow} \mathfrak{c} \stackrel{\pi}{\rightarrow} \mathfrak{g} \rightarrow 0
$$

where $\mathfrak{c}$ and $V$ are Lie algebras with involution and $\iota, \pi$ are morphisms commuting with the respective involutions. 
Now given a Lie triple system $T$, Theorem 6.2.5 provides interpretations of $H_{B H,+}^{n}(T, M)$ for $n$ small. For $M \in T-\operatorname{Mod}$, let $\operatorname{Der}(T, M)$ be the space of linear maps $D: T \rightarrow M$ that satisfy

$$
D([a, b, c])=[D(a), b, c]+[a, D(b), c]+[a, b, D(c)], \quad a, b, c \in T .
$$

Such a map $D$ is inner if there exist $b_{i} \in T$ and $m_{i} \in M$ such that for any $a \in T$,

$$
D(a)=\sum_{i}\left[a, b_{i}, m_{i}\right]
$$

Let $\operatorname{InnDer}(T, M) \subseteq \operatorname{Der}(T, M)$ be the set of such inner derivations. Recall that an ideal of a Lie triple system $T$ is a subspace $A \subset T$ such that $[A, T, T] \subset A$. Also, $T$ is simple if $T$ is not one-dimensional and has no ideals other than 0 and $T$. An ideal $A$ is solvable if the series defined by $A^{(0)}=A, \ldots, A^{(n+1)}=\left[T, A^{(n)}, A^{(n)}\right], \ldots$ is eventually 0 . Then $T$ is semisimple if it has no nonzero solvable ideals. See [19] for more details.

Corollary 6.2.6 ([12]). Let $T$ be a Lie triple system, $M \in T$-Mod, and $N \in$ $K(T)$-Mod.

(a) $H_{B H,+}^{0}(T, M)=0$.

(b) $H_{B H,+}^{1}(T, M) \cong \operatorname{Der}(T, M) / \operatorname{InnDer}(T, M)$.

(c) (Whitehead Lemma) Suppose char $(k)=0$, and that $T$ is semisimple. If $\operatorname{dim}(T)$ and $\operatorname{dim}(M)$ are both finite, then $H_{B H,+}^{1}(T, M)=0=H_{B H,+}^{2}(T, M)$.

(d) Suppose that char $(k)=0$ and $T$ is simple and finite-dimensional. Then $H_{B H,+}^{3}(T, k)=0$ if $L_{u}(T)$ is simple, and $H_{B H,+}^{3}(T, k) \cong k$ if $L_{u}(T)$ is not simple 7

We can use Theorem 6.2.5 to obtain the following result which further compares the functor $j_{*}^{\prime}$ to the functor $j_{*}$ introduced in Definition/Remark 3.8.

Theorem 6.2.7. Let $T$ be an arbitrary Lie triple system and let $M \in T$-Mod. Then $j_{*}^{\prime} M=j_{*} M$ if and only if $\operatorname{Der}(T, M)=\operatorname{InnDer}(T, M)$. In particular, $j_{*}^{\prime} k=$ $j_{*} k$ if and only $T=[T, T, T]$, i.e., if and only if $L_{u}(T)$ is perfect.

Proof. By Theorem 3.9(b), there is a short exact sequence

$$
0 \rightarrow j_{*}^{\prime} M \rightarrow j_{*} M \rightarrow i_{*} V \rightarrow 0
$$

for some $V \in \mathbf{V e c}_{k}$. The long exact sequence for $\operatorname{Ext}_{K(T)}^{\bullet}\left(k^{+},-\right)$gives an exact sequence

$$
\begin{aligned}
\operatorname{Hom}_{K(T)}\left(k^{+}, j_{*} M\right) & \rightarrow \operatorname{Hom}_{K(T)}\left(k^{+}, i_{*} V\right) \rightarrow \operatorname{Ext}_{K(T)}^{1}\left(k^{+}, j_{*}^{\prime} M\right) \\
& \rightarrow \operatorname{Ext}_{K(T)}^{1}\left(k^{+}, j_{*} M\right) \rightarrow \cdots
\end{aligned}
$$

By Theorems 3.9 (a), (c), $\operatorname{Hom}_{K(T)}\left(k^{+}, j_{*} M\right)=\operatorname{Ext}_{K(T)}^{1}\left(k^{+}, j_{*} M\right)=0$. Hence

$$
i_{*} V \cong \operatorname{Hom}_{K(T)}\left(k^{+}, i_{*} V\right) \cong \operatorname{Ext}_{K(T)}^{1}\left(k^{+}, j_{*}^{\prime} M\right) .
$$

By Corollary 6.2.6 (b), $\operatorname{Der}(T, M)=\operatorname{InnDer}(T, M)$ if and only if $H_{B H,+}^{1}(T, M)=$ $\operatorname{Ext}_{K(T)}^{1}\left(k^{+}, j_{*}^{\prime} M\right)=0$. So, $j_{*}^{\prime} M=j_{*} M$ if and only if $\operatorname{Der}(T, M)=\operatorname{InnDer}(T, M)$, as required.

\footnotetext{
${ }^{7}$ Under these hypotheses on $T, 19$ Thm. 2.13] shows that $L_{u}(T)$ must take one of two forms. Namely, either $L_{u}(T)$ is a simple Lie algebra and $T$ is not isomorphic to $\mathfrak{g}_{\text {trip }}$ for any Lie algebra $\mathfrak{g}$, or else $T \cong \mathfrak{g}_{\text {trip }}$ for some simple Lie algebra $\mathfrak{g}$, and $L_{u}(T) \cong \mathfrak{g} \oplus \mathfrak{g}$ with involution $\theta(a, b)=(b, a)$.
} 
Finally, $\operatorname{Der}(T, k)$ is isomorphic to the dual space $(T /[T, T, T])^{*}$, while $\operatorname{InnDer}(T, k)=0$. Since $T /[T, T, T] \cong L_{u}(T) /\left[L_{u}(T), L_{u}(T)\right]$, the last assertion follows.

6.3. Cohomology structure. To begin, observe that when $M=k$, we have $j_{*}^{\prime} M \cong k^{-}$. Thus, we have the isomorphism $H_{B H,-}^{n}(T, k) \cong \operatorname{Ext}_{K(T)}^{n}\left(k^{-}, k^{-}\right)$. For any $N \in K(T)$-Mod, $\operatorname{Ext}_{K(T)}^{\bullet}(N, N)$ is, of course, a $k$-algebra under the Yoneda product. Furthermore, by Lemma 2.6 and the comments following it, $K(T)$ is a (cocommutative) Hopf algebra. Together with [2, Prop. 3.2.1 and Cor. 3.2.2], this implies that $\operatorname{Ext}_{K(T)}^{\bullet}(k, k)$ is a graded-commutative algebra under cup product (equaling the Yoneda product). In addition, the map $-\otimes_{k} k^{-}: \operatorname{Ext}_{K(T)}\left(k^{+}, k^{+}\right) \rightarrow$ $\operatorname{Ext}_{K(T)}^{\bullet}\left(k^{-}, k^{-}\right)$is an isomorphism (see the remarks below).

We summarize these observations in the following proposition.

Proposition 6.3.1. Let $T$ be any Lie triple system, and $N \in K(T)-$ Mod. Then $\operatorname{Ext}_{K(T)}(N, N)$ is a k-algebra under the Yoneda product. In the special case $N=$ $j_{*}^{\prime} k, \operatorname{Ext}_{K(T)}^{\bullet}\left(k^{-}, k^{-}\right) \cong H_{B H,-}^{\bullet}(T, k)$ is a graded-commutative $k$-algebra under cup product, and the cup product agrees with the Yoneda product.

Example 6.3.2. Return to the situation of Example 6.2.1 Let $\mathfrak{p}$ be the -1 eigenspace of $\theta$ on $\mathfrak{g}$. Then

$$
H_{B H, \pm}^{\bullet}(\mathfrak{p}, k) \cong H_{ \pm}^{\bullet}\left(\mathfrak{g}, k^{+}\right) .
$$

These are described in Example 6.2.1

For a Lie triple system $T$, the bialgebra structure on $K(T)$ allows us to consider the functor

$$
\nu: K(T)-\operatorname{Mod} \rightarrow K(T)-\mathbf{M o d}, \quad N \mapsto k^{-} \otimes N .
$$

As a $K(T)$-module, $\nu(N)$ has the same action of $U(T)$, but now $\theta$ acts as $-\theta$. Clearly, $\nu$ is an equivalence of categories. For the idempotents $f, e \in K(T)$ as in (3.11), it is easy to check that $\psi: \nu(K(T) e) \rightarrow K(T) f$ defined by $\psi(a e)=-a f$ determines an isomorphism of $K(T)$-modules (just verify that $\psi$ and $\theta$ commute, using $\theta(e)=-e$ and $\theta(f)=f)$. Likewise, $\nu(K(T) f) \cong K(T) e$, since $\nu^{2}=\mathrm{id}$. Using this, we see that for $Y_{n}^{ \pm}$as in (6.2.2), $\nu\left(Y_{n}^{+}\right)=Y_{n}^{-}$(resp., $\left.\nu\left(Y_{n}^{-}\right)=Y_{n}^{+}\right)$. We thus obtain isomorphisms

$$
H_{+}^{n}\left(L_{u}(T), N\right) \cong H_{-}^{n}\left(L_{u}(T), \nu(N)\right)
$$

for any $N \in K(T)-$ Mod.

Proposition 6.3.5. Let $T$ be any Lie triple system and $M \in T$-Mod. Then

$$
H_{B H,-}^{\bullet}(T, M) \text { and } H_{B H,+}^{\bullet}(T, M)
$$

are naturally modules for the algebra $H_{B H_{,-}}^{\bullet}(T, k)$.

Proof. By definition, $H_{B H,-}^{\bullet}(T, M)=\operatorname{Ext}_{K(T)}^{\bullet}\left(k^{-}, j_{*}^{\prime} M\right)$; so it has a natural module structure for $H_{B H,-}^{\bullet}(T, k) \cong \operatorname{Ext}_{K(T)}^{\bullet}\left(k^{-}, k^{-}\right)$. Next,

$$
H_{B H,+}^{\bullet}(T, M) \cong \operatorname{Ext}_{K(T)}^{\bullet}\left(k^{+}, j_{*}^{\prime} M\right) \cong \operatorname{Ext}_{K(T)}^{\bullet}\left(k^{-}, \nu\left(j_{*}^{\prime} M\right)\right) ;
$$

so it also has a natural $H_{B H,-}^{\bullet}(T, k)$-module structure. 
6.4. Relating the cohomology theories. Let $T$ be a Lie triple system. This subsection provides some comparison between the cohomology groups $H^{\bullet}(T, M)$ and the groups $H_{B H, \pm}^{\bullet}(T, M)$ discussed in the previous section.

Proposition 6.4.1. Let $T$ be a Lie triple system and let $M, N \in T$-Mod. Suppose that $M^{\prime} \in K(T)$-Mod satisfies $j^{*} M^{\prime} \cong M$. Then there is a first quadrant spectral sequence

$$
E_{2}^{m, n}=\operatorname{Ext}_{K(T)}^{m}\left(M^{\prime}, R^{n} j_{*} N\right) \Rightarrow \operatorname{Ext}_{T}^{m+n}(M, N) .
$$

If $\operatorname{dim} R^{n} j_{*} N=d_{n}$, then

$$
E_{2}^{m, n} \cong \operatorname{Ext}_{K(T)}^{m}\left(M^{\prime}, k^{+}\right)^{\oplus d_{n}}
$$

for all $n>0$. Finally, $E_{2}^{m, n}=0$ for $m>\operatorname{dim} L_{u}(T)$.

Proof. First,

$$
\operatorname{Hom}_{T}(M,-) \cong \operatorname{Hom}_{K(T)}\left(M^{\prime}, j_{*}-\right) ;
$$

so the functor $h_{M}=\operatorname{Hom}_{T}(M,-)$ factors as $h_{M}=h_{M^{\prime}} \circ j_{*}$, where $h_{M^{\prime}}=$ $\operatorname{Hom}_{K(T)}\left(M^{\prime},-\right): K(T)-\mathbf{M o d} \rightarrow \mathbf{V e c}_{k}$. Since $j_{*}$ has an exact left adjoint $j^{*}$, $j_{*}$ carries injective $T$-modules to injective $K(T)$-modules. Thus, the spectral sequence can be taken to be the Grothendieck spectral sequence associated to the above factorization of $\operatorname{Hom}_{T}(M,-)$.

That $E_{2}^{m, n} \cong \operatorname{Ext}_{K(T)}^{m}\left(M^{\prime}, k^{+}\right)^{\oplus d_{n}}$ for $n>0$ follows from Theorem 3.9(d), which asserts that $R^{n} j_{*} N$ is a trivial $K(T)$-module. The final assertion is clear since the discussion in $\$ 6.2$ implies that $K(T)$ has homological dimension at most $\operatorname{dim} L_{u}(T)$.

Remark 6.4.2. If $T$ is finite-dimensional with $r=\operatorname{dim} T$, then $\operatorname{dim} L_{u}(T) \leq r+$ $r(r-1) / 2$. Thus, in the spectral sequence of Proposition 6.4.1 certainly $E_{2}^{m, n}=0$ for $m>r+r(r-1) / 2$.

In practice, we can take $M^{\prime}=j_{*}^{\prime} M, j_{*} M$, or even $j_{!} M$. In particular, we have the following result:

Corollary 6.4.3. Let $T$ be a Lie triple system and $N \in T-$ Mod.

(a) There is a spectral sequence

$$
E_{2}^{m, n}=\operatorname{Ext}_{K(T)}^{m}\left(k^{-}, R^{n} j_{*} N\right) \Rightarrow H^{m+n}(T, N)
$$

in which $E_{2}^{m, 0}=H_{B H,-}^{m}(T, N)$.

(b) The edge map $E_{2}^{\bullet, 0} \rightarrow H^{\bullet}(T, N)$ induces a natural map $H_{B H,-}^{\bullet}(T, N) \rightarrow$ $H^{\bullet}(T, N)$.

Proof. (a) This follows from Proposition 6.4.1 since $j_{*}^{\prime} k \cong k^{-}$satisfies $j^{*} k^{-} \cong k$.

(b) Since $E_{2}^{\bullet, 0}=\operatorname{Ext}_{K(T)}^{\bullet}\left(k^{-}, j_{*} N\right)$, the map $j_{*}^{\prime} N \rightarrow j_{*} N$ provided in Theorem 3.9 gives a map

$$
H_{B H,-}^{\bullet}(T, N)=\operatorname{Ext}_{K(T)}^{\bullet}\left(k^{-}, j_{*}^{\prime} N\right) \rightarrow \operatorname{Ext}_{K(T)}^{\bullet}\left(k^{-}, j_{*} N\right),
$$

which when composed with the edge map gives a map $H_{B H,-}^{\bullet}(T, N) \rightarrow H^{\bullet}(T, N)$ as required.

Since $\operatorname{Ext}_{K(T)}^{1}\left(k^{+}, j_{*}^{\prime} M\right)=H_{B H,+}^{1}(T, M)$, Theorem 6.2.6(c) and Theorem 3.9 imply the following result, recalling that $K(T)$ - mod denotes the category of finitedimensional $K(T)$-modules. 
Corollary 6.4.4. Suppose $T$ is a finite-dimensional, semisimple Lie triple system and char $(k)=0$. Then $j_{*}^{\prime}=j_{*}$ as functors on $M \in K(T)$-mod.

Remark 6.4.5. We will give a simple example to show that, in general, $H^{\bullet}(T, M) \nsucceq$ $H_{B H,-}^{\bullet}(T, M)$. Consider the 2-dimensional trivial Lie triple system $T$. Let $\mathfrak{g}=$ $L_{u}(T)=T \oplus \bigwedge^{2} T$. Then $\mathfrak{g}$ has basis $x, y, z=x \wedge y$ in which $z$ is central, $\theta(x)=-x$, $\theta(y)=-y$, and $\theta(z)=z$. The $\theta$-module $H^{\bullet}(\mathfrak{g}, k)$ can be computed directly as a $\theta$-module: If $d_{ \pm}^{i}=\operatorname{dim} H_{ \pm}^{i}\left(\mathfrak{g}, k^{+}\right)$, then

$$
\begin{aligned}
d_{-}^{1} & =d_{-}^{2}=2 ; \\
d_{+}^{0} & =d_{+}^{3}=1 ; \\
d_{+}^{1}=d_{+}^{2} & =d_{-}^{0}=d_{-}^{3}=0 .
\end{aligned}
$$

It follows that $H_{B H,-}^{1}(T, k)=\operatorname{Ext}_{K(T)}^{1}\left(k^{-}, k^{-}\right)=0$. Now consider $H^{1}(T, k)$. In the spectral sequence Corollary 6.4.3 for $N=k$, we have

$$
E_{2}^{0,1}=\operatorname{Hom}_{K(T)}\left(k^{-}, R^{1} j_{*} k\right)=0
$$

by Theorem 3.9(e). Thus,

$$
H^{1}(T, k) \cong E_{2}^{1,0}=\operatorname{Ext}_{K(T)}^{1}\left(k^{-}, j_{*} k\right) .
$$

Since $d_{-}^{2}=2$, there is an exact sequence

$$
0 \rightarrow k^{-} \rightarrow j_{*} k \rightarrow i_{*} V \rightarrow 0
$$

in $K(T)$-Mod, where $\operatorname{dim} V=2$. The long exact sequence of $\operatorname{Ext}_{K(T)}\left(k^{-},-\right)$and the vanishing of $\operatorname{Ext}_{K(T)}^{1}\left(k^{-}, k^{-}\right)$imply that $H^{1}(T, k)=\operatorname{Ext}_{K(T)}^{1}\left(k^{1}, j_{*} k\right)$ is the kernel of a map

$$
\operatorname{Ext}_{K(T)}^{1}\left(k^{-}, V\right) \rightarrow \operatorname{Ext}_{K(T)}^{2}\left(k^{-}, k^{-}\right) .
$$

But $\operatorname{dim} \operatorname{Ext}_{K(T)}^{1}\left(k^{-}, V\right)=2 d_{-}^{2}=4$ while $\operatorname{dim} \operatorname{Ext}_{K(T)}^{2}\left(k^{-}, k^{-}\right)=d_{+}^{2}=0$. Thus, $\operatorname{dim} H^{1}(T, k)=4$, while $\operatorname{dim} H_{B H_{-}}^{1}(T, k)=\operatorname{dim} \operatorname{Ext}_{K(T)}^{1}\left(k^{-}, k^{-}\right)=0$.

6.5. Restricted cohomology and the Hochschild maps. So far, we have confined attention to the cohomology in the category $T-$ Mod. Now assume that $T$ is a restricted Lie triple system over a field $k$ of positive characteristic $p \neq 2$ as defined in $\S 4$. If $M, N \in T-\operatorname{Mod}_{[p]}$, let $\operatorname{Ext}_{T}^{\bullet}(M, N)_{[p]}$ denote the corresponding Ext-groups computed in the category $T-\mathbf{M o d}_{[p]}$ of restricted $T$-modules. By Theorem 5.4 these groups can be computed in the standard way using either an injective resolution of $N$ or a projective result of $M$. We can also consider the groups $H_{[p]}^{\bullet}(T, M)=$ $\operatorname{Ext}_{T}^{\bullet}(k, M)_{[p]}$ for $M \in T-\operatorname{Mod}_{[p]}$. In addition, Theorem 5.4 provides a means to

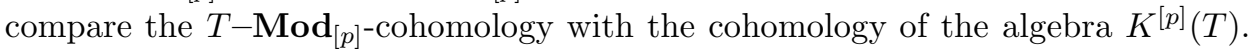
For example, by analogy with Proposition 6.4.1 for any $M, N \in T-\mathbf{M o d}_{[p]}$, if $M^{\prime} \in K^{[p]}(T)$-Mod satisfies ${ }^{[p]} j^{*} M^{\prime} \cong M$, there is a first quadrant spectral sequence with $E_{2}^{m, n}=\operatorname{Ext}_{K[p](T)}^{m}\left(M^{\prime}, R^{n[p]} j_{*} N\right)$ which converges to $\operatorname{Ext}_{T}^{\bullet}(M, N)_{[p]}$. Consequently, setting $H_{[p],-}^{m}(T, N)=\operatorname{Ext}_{K^{[p]}(T)}^{m}\left(k^{-},{ }^{[p]} j_{*}^{\prime} N\right)$ (the restricted analogue of the Harris cohomology group $H_{B H,-}^{m}(T, N)$ of Definition 6.2.4), we have, just as in Corollary 6.4.3 a spectral sequence $E_{2}^{m, n}=\operatorname{Ext}_{K^{[p]}(T)}^{m}\left(k^{-}, R^{n[p]} j_{*} N\right)$ 
$\Rightarrow H_{[p]}^{m+n}(T, N)$ in which $E_{2}^{m, 0}=H_{[p],-}^{m}(T, N)$. The edge map then gives rise to a natural map

$$
H_{[p],-}^{\bullet}(T, N) \rightarrow H_{[p]}^{\bullet}(T, N)
$$

We conclude this paper with some brief remarks concerning "Hochschild maps" first used in [7 to compute the restricted cohomology of restricted Lie algebras of classical type 8 Consider the Lie algebra $L_{u}^{[p]}(T)$ introduced in Definition 4.8. Let $H=[T, T] \subseteq L_{u}^{[p]}(T)$, so that $H$ is a subalgebra of the Lie algebra $L_{u}^{[p]}(T)$ and $L_{u}^{[p]}(T)=T \oplus H$. Let $\theta \in \operatorname{Aut}\left(L_{u}^{[p]}(T)\right)$ be the involution satisfying $\left.\theta\right|_{T}=-$ id and $\left.\theta\right|_{H}=+\mathrm{id}$. Now let $\phi \in T^{*}$, and form the split central extension $\widetilde{L_{u}^{[p]}(T)}=$ $L_{u}^{[p]}(T) \oplus k$. For $(X, \lambda) \in \widetilde{T}=T \oplus k$, define

$$
(X, \phi)^{[p]}=\left(X^{[p]}, \phi(X)^{p}\right) .
$$

Thus, for $x \in \widetilde{T}$, ad $x^{[p]}=(\operatorname{ad} x)^{p}$. If $\phi \neq 0, \theta$ extends to an automorphism of $\widetilde{L_{u}^{[p]}(T)}$ satisfying $\theta(x)^{[p]}=\theta\left(x^{[p]}\right)$ for all $x \in \widetilde{T}$, provided that $\theta(X, \lambda)=(\theta(X),-\lambda)$ for all $X \in L_{u}^{[p]}(T), \lambda \in k$. We obtain an extension

$$
0 \rightarrow k \rightarrow \widetilde{T} \rightarrow T \rightarrow 0
$$

of restricted Lie triple systems, in which, as ordinary Lie triple systems, we have $\widetilde{T}=T \oplus k$. In addition, following the construction in Definition 4.14 we obtain an extension

$$
0 \rightarrow k^{-} \rightarrow \mathfrak{A} \rightarrow U^{[p]}(T) \rightarrow 0
$$

of augmented algebras.

An extension $0 \rightarrow A \rightarrow \widehat{\mathfrak{g}} \rightarrow \mathfrak{g} \rightarrow 0$ of Lie algebras in which $A$ is abelian naturally endows $A$ with the structure of a $\mathfrak{g}$-module. If $\mathcal{M}(\mathfrak{g}, A)$ denotes the set of equivalence classes (in an obvious sense) of such extensions of $\mathfrak{g}$ by $A$, then a fundamental result provides a bijection between $\mathcal{M}(\mathfrak{g}, A)$ and the cohomology group $H^{2}(\mathfrak{g}, A)$; see [13, Thm. 3.3, p. 238]. (Furthermore, if $\mathfrak{g}$ is a restricted Lie algebra, an extension $0 \rightarrow A \rightarrow \widehat{\mathfrak{g}} \rightarrow \mathfrak{g} \rightarrow 0$ is said to be restricted if $\widehat{\mathfrak{g}}$ is a restricted Lie algebra in which $A$ sits as a restricted ideal inside the kernel of the restricted homomorphism $\widehat{\mathfrak{g}} \rightarrow \mathfrak{g}$. Letting $H_{[p]}^{2}(\mathfrak{g}, k)$ denote the restricted cohomology group of $\mathfrak{g}$, there likewise exists a bijection between $H_{[p]}^{2}(\mathfrak{g}, k)$ and equivalence classes of restricted extensions $\mathcal{M}_{[p]}(\mathfrak{g}, k)$ of $\mathfrak{g}$ by $k$.) In the context of Lie algebras with involution, this result is extended in Theorem6.2.5(c) above. In a similar way an extension (6.5.8) of augmented algebras corresponds to an element in $\operatorname{Ext}_{K[p](T)}^{2}\left(k^{+}, k^{-}\right)$. In summary, we have defined a natural linear map $\Psi: T^{*(1)} \rightarrow \operatorname{Ext}_{K^{[p]}(T)}^{2}\left(k^{+}, k^{-}\right)$. Here $T^{*(1)}$ denotes the vector space obtained from the underlying abelian group structure on $T^{*}$ by making $\lambda \in k$ act by scalar multiplication by $\lambda^{1 / p}$. We call $\Psi$ the Hochschild map defined by the restricted Lie triple system $T$. It is completely analogous to a similar map $\mathfrak{g}^{*(1)} \rightarrow H_{[p]}^{2}(\mathfrak{g}, k)$; see 7].

Finally, given any element $\zeta \in \operatorname{Ext}_{K^{[p]}(T)}^{2 m}\left(k^{-}, k^{+}\right)$(with $m$ any positive integer), the cup product defines a map $T^{*(1)} \rightarrow \operatorname{Ext}_{K^{[p]}(T)}^{2(m+1)}\left(k^{-}, k^{-}\right), \phi \mapsto \Psi(\phi) \cup \zeta$.

${ }^{8}$ We do not discuss in this paper any possible version of the so-called May spectral sequence (used, e.g., in [7]). 
Since $K^{[p]}(T)$ is a cocommutative Hopf algebra, the algebra $\operatorname{Ext}_{K^{[p]}(T)}^{2 \bullet}\left(k^{-}, k^{-}\right)$is commutative. Finally, applying $j^{*}$ gives a homomorphism

$$
S\left(\Psi_{\zeta}\right): S\left(T^{*}\right)^{(1)} \rightarrow H_{[p],-}^{2 \bullet}(T, k)
$$

from the symmetric algebra with "twisted" scalar multiplication to the cohomology algebra given by $H_{[p],-}^{2 \bullet}(T, k)=\operatorname{Ext}_{K^{[p]}(T)}^{2 \bullet}\left(k^{-}, k^{-}\right)$. Composing (6.5.9) with the natural map $H_{[p],-}^{2 \bullet}(T, k) \rightarrow H_{[p]}^{2 \bullet}(T, k)$ arising from (6.5.6) yields another Hochschild homomorphism

$$
S\left(\Psi_{\zeta}\right): S\left(T^{*}\right)^{(1)} \rightarrow H_{[p]}^{2 \bullet}(T, k)
$$

We will return to these matters in a future paper.

\section{REFERENCES}

[1] A. Beilinson, J. Bernstein, and P. Deligne, Faisceaux pervers, Analyse et topologie sur les espaces singulières, I (Luminy, 1981), Astérisque 100, 1982, pp. 5-171. MR 86g:32015

[2] D. J. Benson, Representations and Cohomology I, Cambridge University Press, Cambridge, 1991. MR 92m:20005

[3] A. Borel and T. A. Springer, Rationality properties of linear algebraic groups, II, Tôhoku Math. Jour. 20 (1968), 443-497. MR 39:5576

[4] J. M. Casas, J.-L. Loday, and T. Pirashvili, Leibniz n-Algebras, Forum Math. 14 (2002), 189-207.

[5] M. Demazure and P. Gabriel, Groupes algébriques, Tome I: Géométrie algébrique, généralités, groupes commutatifs, North-Holland, Amsterdam, 1970. MR 46:1800

[6] C. Faith, Algebra: Rings, modules and categories I, Springer-Verlag, New York, 1973. MR 51:3206

[7] E. Friedlander and B. Parshall, Cohomology of Lie Algebras and Algebraic Groups, Amer. Jour. Math. 108 (1986), 235-253. MR 87c:20080

[8] E. Friedlander and B. Parshall, Geometry of p-Unipotent Lie Algebras, Jour. Algebra 109 (1987), 25-45. MR 89a:17017

[9] E. Friedlander and B. Parshall, Support varieties for restricted Lie algebras, Invent. Math. 86 (1986), 553-563. MR 88f:17018

[10] E. Friedlander and B. Parshall, Modular representation theory of Lie algebras, Amer. Jour. Math. 110 (1988), 1055-1094. MR 89j:17015

[11] W. Greub, S. Halperin, and R. Vanstone, Connections, Curvature and Cohomology, III, Academic Press, New York, 1976. MR 53:4110

[12] B. Harris, Cohomology of Lie Triple Systems and Lie Algebras with Involution, Trans. Amer. Math. Soc. 98 (1961), 148-162. MR 22:11068

[13] P. Hilton and U. Stammbach, A Course in Homological Algebra, Springer-Verlag, New York, 1997. MR 97k:18001

[14] T. L. Hodge, Lie Triple Systems, Restricted Lie Triple Systems, and Algebraic Groups, Jour. Algebra 244 (2001), 533-580.

[15] N. Jacobson, Lie and Jordan Triple Systems, Amer. Jour. Math. 71 (1949), 149-170. MR 10:426c

[16] N. Jacobson, General Representation Theory of Jordan Algebras, Trans. Amer. Math. Soc. 70 (1951), 509-530. MR 12:797d

[17] N. Jacobson, Lie Algebras, Dover, New York, 1962. MR 26:1345

[18] N. Kamiya and S. Okubo, On triple systems and Yang-Baxter equations, Proceedings of the Seventh International Colloquium on Differential Equations (Plovdiv, 1996), 189-196, VSP, Utrecht, 1997. MR 98m:17007

[19] W. G. Lister, A Structure Theory of Lie Triple Systems, Trans. Amer. Math. Soc. 72 (1952), 217-242. MR 13:619d

[20] O. Loos, Symmetric Spaces, vol. I, W. A. Benjamin, New York, 1969. MR 39:365a

[21] S. Mac Lane, Categories for the Working Mathematician, Springer-Verlag, 1971. MR 50:7275

[22] G. P. Nagy, Algebraische kommutative Moufang-Loops (prefinal version), Doctoral Dissertation, Friedrich-Alexander-Universität Erlangen-Nürnberg, 2000. 
[23] D. Nakano, B. Parshall and D. Vella, Support varieties for algebraic groups, Jour. reine angew. Math. 547 (2002), 15-49.

[24] D. Quillen, Homotopical algebra, Springer-Verlag Lectures Notes in Mathematics, vol. 43, New York, 1967. MR 36:6480

[25] R. Richardson, Orbits, invariants, and representations associated to involutions of reductive groups, Invent. Math. 66 (1982), 287-312. MR 83i:14042

[26] J. D. H. Smith, Representation theory of infinite groups and finite quasigroups, Séminaire de mathématiques supérieures, Université de Montréal, Montréal, 1986. MR 88a:20088

[27] A.A. Suslin, E.M. Friedlander, C.P. Bendel, Infinitesimal one-parameter subgroups and cohomology, Jour. Amer. Math. Soc., 10 (1997), 693-728. MR 98h:14055b

[28] A.A. Suslin, E.M. Friedlander, C.P. Bendel, Support varieties for infinitesimal group schemes, Jour. Amer. Math. Soc., 10 (1997), 729-759. MR 98h:14055c

[29] K. Yamaguti, On the cohomology space of Lie triple systems, Kumamoto J. Sci. Ser. A, 5 (1960), 44-52. MR 24:A2606

[30] K. Yamaguti, On Weak Representations of Lie Triple Systems, Kumamoto J. Sci., Ser. A. 8 (1968), 107-114. MR 40:7324

Department of Mathematics, Western Michigan University, Kalamazoo, Michigan 49008

E-mail address: terrell.hodge@wmich.edu

Department of Mathematics, University of Virginia, Charlottesville, Virginia 22903

E-mail address: bjp8w@virginia.edu 